Review Article

\title{
A Preclinical Systematic Review of Ginsenoside-Rg1 in Experimental Parkinson's Disease
}

\author{
Liang Song, ${ }^{1,2}$ Meng-Bei Xu, ${ }^{1}$ Xiao-Li Zhou, ${ }^{1}$ Dao-pei Zhang, ${ }^{2}$ \\ Shu-ling Zhang, ${ }^{2}$ and Guo-qing Zheng ${ }^{1}$ \\ ${ }^{1}$ Department of Neurology, The Second Affiliated Hospital and Yuying Children's Hospital of Wenzhou Medical University, \\ Wenzhou, China \\ ${ }^{2}$ Department of Neurology, Zhengzhou People's Hospital, Zhengzhou 450003, China
}

Correspondence should be addressed to Shu-ling Zhang; zs166317@163.com and Guo-qing Zheng; gq_zheng@sohu.com

Received 23 November 2016; Revised 24 January 2017; Accepted 14 February 2017; Published 13 March 2017

Academic Editor: Elena Azzini

Copyright (C) 2017 Liang Song et al. This is an open access article distributed under the Creative Commons Attribution License, which permits unrestricted use, distribution, and reproduction in any medium, provided the original work is properly cited.

\begin{abstract}
To date, no drug has been proven to be neuroprotective or disease-modifying for Parkinson's disease (PD) in clinical trials. Here, we aimed to assess preclinical evidence of Ginsenosides-Rg1 (G-Rg1), a potential neuroprotectant, for experimental PD and its possible mechanisms. Eligible studies were identified by searching six electronic databases from their inception to August 2016. Twenty-five eligible studies involving 516 animals were identified. The quality score of these studies ranged from 3 to 7 . Compared with the control group, two out of the 12 studies of MPTP-induced PD showed significant effects of G-Rg1 for improving the rotarod test $(P<0.01)$, two studies for improving the swim-score values $(P<0.01)$, six studies for improving the level of TH protein expression $(P<0.01)$, and two studies for increasing the expression of TH mRNA in the substantia nigra of mice $(P<$ $0.01)$. The studies reported that G-Rg1 exerted potential neuroprotective effects on PD model through different mechanisms as antineuroinflammatory activities $(n=10)$, antioxidant stress $(n=3)$, and antiapoptosis $(n=11)$. In conclusion, G-Rg1 exerted potential neuroprotective functions against PD largely by antineuroinflammatory, antioxidative, and antiapoptotic effects. G-Rg1 as a promising neuroprotectant for PD needs further confirmation by clinical trials.
\end{abstract}

\section{Introduction}

Parkinson's disease (PD) is the second most frequent neurodegenerative disease after Alzheimer's disease characterized by the loss of dopamine-containing cells in the substantia nigra (SN) [1]. The clinical symptoms of PD are a wealth of motor symptoms and nonmotor symptoms. The treatment is divided into two directions: symptomatic therapy for motor symptoms and modifying the underlying disease process through neuronal protection or restoration. However, current treatments of PD are mainly symptomatic therapies and no treatment has yet been proven to be truly neuroprotective [2]. Dopamine replacement therapy (L-DOPA and dopamine agonists) is still the most effective symptomatic treatment of $\mathrm{PD}$, but this treatment frequently induces therapy-related motor complications such as dyskinesia, choreoathetosis, and fluctuations in motor function [3]. Thus, a number of PD patients resort to various kinds of complementary or alternative medicine (CAM) to improve their motor and/or nonmotor symptoms [4]. Traditional Chinese medicine (TCM), as one of the most important parts in CAM, has played a vital role in the medical care of PD patients for thousands of years [5]. Ginseng, the root of Panax species (C.A. Meyer Araliaceae), is a well-known traditional Chinese herbal medicine that has been used for various kinds of diseases in China, Japan, and Korea for thousands of years and is still a popularly and worldwide used natural medicine in modern time [6]. The major pharmacologically active ingredients of ginseng are Ginsenosides and they are responsible for most of the activities of ginseng [7]. Ginsenosides are divided into two categories as follows: (1) the protopanaxadiol (PPD) type: $\mathrm{Rb} 1, \mathrm{Rb} 2, \mathrm{Rb} 3, \mathrm{Rc}, \mathrm{Rd}$, and $\mathrm{Rg} 3$; (2) the protopanaxatriol (PPT) type: Rg1, Re, Rf, and Rg2 [8]. It has been reported that Ginsenosides-Rg1 (G-Rg1) might have neuroprotective 
effects and little toxicity both in vitro and in vivo [9]. It also has beneficial effects on many neurological conditions, including the progressive neurodegenerative diseases such as PD [10]. The mechanisms of the neuroprotective effect of G-Rg1 include potentiating nerve growth factors, increasing anti-inflammation, antioxidation, and antiapoptosis, inhibiting excite toxicity and $\mathrm{Ca}^{2+}$ overinflux into neurons, maintaining cellular ATP levels, and preserving structural integrity of neurons [11]. However, no systematic review has been conducted to assess the effect of G-Rgl on experimental PD models to date. Systematic review of all available evidence from animal experiments before clinical trials can provide us adequate interpretation of the limitations and potential of a novel treatment strategy [12]. Therefore, in the present study, we conducted a systematic review of all available animal studies to evaluate the preclinical evidence of G-Rg1 for experimental PD.

\section{Methods}

2.1. Search Strategy. Two trained researchers independently searched studies on the effects of G-Rg1 on PD from their inception to August 2016 in the following databases: PubMed, the Cochrane Database, Excerpta Medica (EMBASE), Chinese National Knowledge Infrastructure (CNKI), Wanfang database, and VIP Information Database. The following search terms were used: (Ginseng OR Ginsenoside OR Ginsenoside-Rg1 OR G-Rg1 OR Ginseng saponin) AND (Parkinson disease OR Parkinson's disease OR PD) in both English and Chinese.

2.2. Inclusion Criteria. Studies that were included met all of the following criteria: (1) all studies should test the effect of G-Rg1 on animal models of PD, regardless of language, blinding, or publication status; (2) in the treatment group, any intervention that used G-Rg1 for PD should be included irrespective of the frequency, dose, the method of injection, and intensity; (3) in the control group, animals were treated with normal saline or nothing.

2.3. Exclusion Criteria. Prespecified exclusion criteria were the following: (1) not reporting the efficacy of G-Rg1 on animal experiment of PD; (2) another neuroprotective agent being administered in the treatment group in addition to $\mathrm{G}$ Rg1; (3) no control group; (4) reviews, case reports, abstracts, letters, comments, study protocol, editorials, and clinical guidelines; (5) duplicate publication.

2.4. Outcome Measurements. The primary outcome of the interest was the behavioral assessments, including rotarod test, pole test, wire suspension test, and the values of swimscore. Secondary outcomes were the number of Tyrosine Hydroxylase- (TH-) positive dopamine neurons in the substantia nigra pars compacta (SNpc), levels of $\mathrm{TH}$ protein expression in the SNpc, and the mechanisms of G-Rg1.

2.5. Data Extraction. Two investigators independently extracted information from each study, including (1) the first author's name and publication year; (2) individual data obtained for experimental animals including species, sex, number, weight, and anesthetic used; (3) experimental model; (4) information on treatment group including route of administration, dosage, and time for treatment; (5) data of control groups extracted as well as route of administration, dosage, and time of administration; (6) intergroup difference of each outcome measure; (7) outcome measures, including the behavioral exhibition of PD animal models, the number of $\mathrm{TH}$ neurons, and possible mechanisms of neuroprotective effects of G-Rg1 against PD. If outcomes were presented at different time points, data from the last time point were extracted. If the outcome data for metaanalysis were only expressed graphically or missing, we made attempt to contact authors for further information. When a response was not received, we used digital ruler software to measure the data from the graphs. We extracted data of mean value and standard deviation for each comparison from every study. Any disagreements were resolved through consultation with a corresponding author (Guo-qing Zheng).

2.6. Quality Assessment. The methodological quality of the included studies was assessed based on a nine-item modified scale from the Collaborative Approach to Meta-Analysis and Review of Animal Data from Experimental Studies (CAMARADES) [13]. The modified CAMARADES includes the following criteria: (1) peer reviewed publication; (2) control of temperature; (3) random allocation to groups; (4) blinded assessment of behavioral outcome; (5) use of anesthetic without significant intrinsic neuroprotective activity; (6) calculation of the sample size necessary to achieve sufficient power; (7) appropriate animal model which uses animals without relevant comorbidities (aged, diabetic, or hypertensive); (8) compliance with animal welfare regulations; (9) statement of potential conflict of interests. For quality assessment score, the interquartile range of score across studies was reported.

2.7. Statistical Analysis. We conducted statistical analysis using Cochrane's Review Manager (version 5.3) software. Data extracted from each study were considered as continuous data. WMD (weighted mean difference) is a standard statistic that measures the absolute difference between the mean values in two groups. Meanwhile, standardized mean difference (SMD) is also used as a summary statistic in metaanalysis when all the studies assess the same outcome but measure it in a variety of ways [14]. Heterogeneity among studies was estimated using Cochran's $Q$ test (reported with $\chi^{2}$ value and $P$ value) and $I^{2}$ statistic. $I^{2}$ values of 75,50 , and $25 \%$ correspond to high, medium, and low levels of heterogeneity, respectively; $I^{2}$ values less than $50 \%$ indicated an acceptable degree of heterogeneity between studies [15]. Probability values of 0.05 were considered significant. Sensitivity analyses omitting each study at a time from the original analysis were conducted to verify our main results to be robust. 


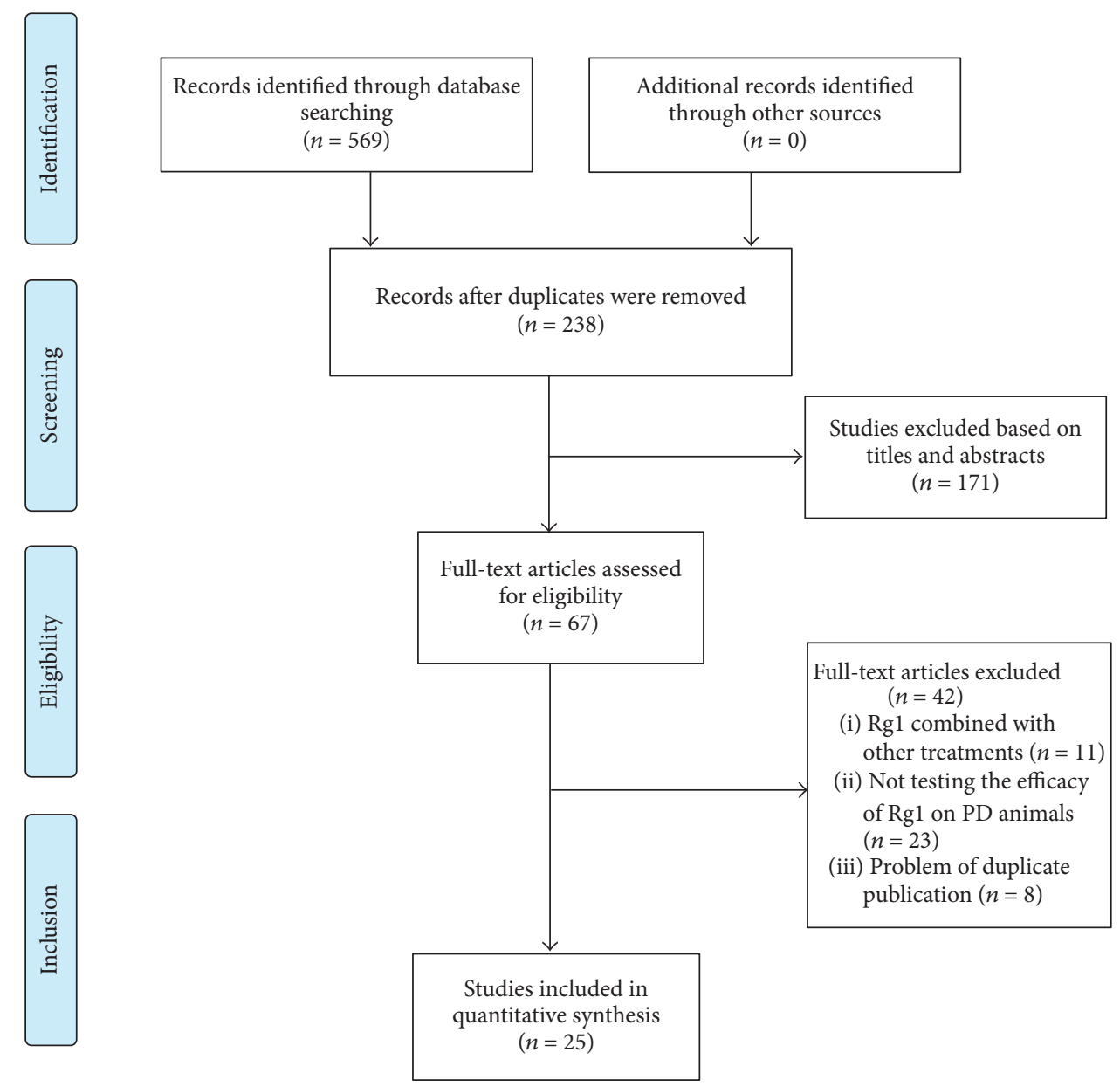

FIGURE 1: Summary of the process for identifying candidate studies.

\section{Results}

3.1. Description of Studies. We identified 569 potentially relevant articles from six electronic databases. After removing duplicates, 238 references remained. Through screening titles and abstracts, 171 studies were excluded. After full-text evaluation on the remaining 67 articles, 11 articles were removed because of combination with other treatment drugs in the experimental group; 23 articles were excluded because they did not test the efficacy of G-Rg1 on PD animals; 8 articles were excluded because of duplicate publication. Eventually, 25 eligible studies [16-40] were identified (Figure 1).

3.2. Study Characteristics. The 25 eligible studies included 516 animals from two species: 415 C57BL/6 mice and 111 ovariectomized Wistar rats. The weight of $\mathrm{C} 57 \mathrm{BL} / 6$ mice varied from $16 \mathrm{~g}$ to $30 \mathrm{~g}$, and the weight of ovariectomized Wistar rats varied from $200 \mathrm{~g}$ to $250 \mathrm{~g}$. Eight articles [1620, 35, 39, 40] were published in English academic journals and 17 articles [21-34, 36-38] were published in Chinese academic journals from 2001 to 2016 . As for experimental animal model, twenty studies used 1-methyl-4-phenyl-1,2,3,6tetrahydropyridine- (MPTP-) induced PD model [16-34,
39], 4 studies [35-38] used 6-hydroxydopamine (6-OHDA) induced PD model, and 1 study used lipopolysaccharide(LPS-) induced PD model [40]. In terms of gender, five studies $[25,35,37,38,40]$ used merely female animals and eighteen studies [16, 18-20, 22-24, 26-34, 36, 39] used merely male animals, while the remaining 2 studies [17, 21] did not report gender. Among 25 included studies, one study used ether cotton balls to induce anesthesia [33], two studies used urethane $[17,26]$, eleven studies used chloral hydrate $[16,23$, $27,28,30,32,35-38,40]$, four studies used pentobarbital sodium $[18,20,31,39]$, three studies did not report anesthesia $[24,29,34]$, and the remaining four studies did not report the method of executing the animals [19, 21, 22, 25]. For all included studies, the intervention measures for experimental groups were injection with G-Rgl before injection of MPTP, 6-OHDA, or LPS. Fifteen studies used behavioral assessments as primary outcomes [16, 17, 22-24, 26, 28, 29, 32-37, 39]. TH-positive dopamine neurons in the SNpc were observed in 16 studies [16-20, 24, 26, 28-32, 34, 35, 38, 39]. Nine studies reported the levels of $\mathrm{TH}$ protein expression $[16,18,22-$ $24,29,30,32,34]$. Meanwhile, the indexes related to the mechanisms of G-Rg1 were used as outcomes as the antiinflammatory activities in 10 studies [16-18, 21-23, 29, 33, 


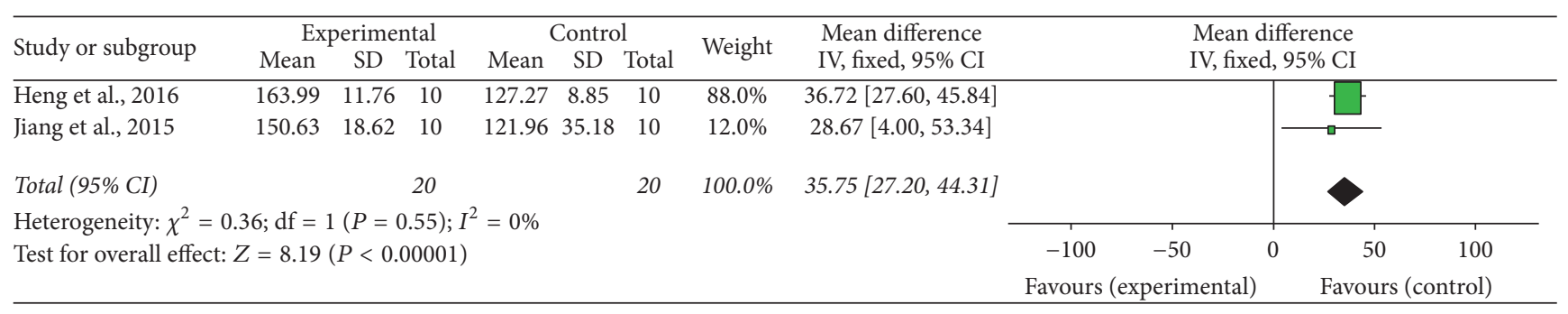

FIGURE 2: The forest plot: effects of G-Rg1 for improving the rotarod test compared with control group. Note: G-Rg1: Ginsenosides-Rg1.

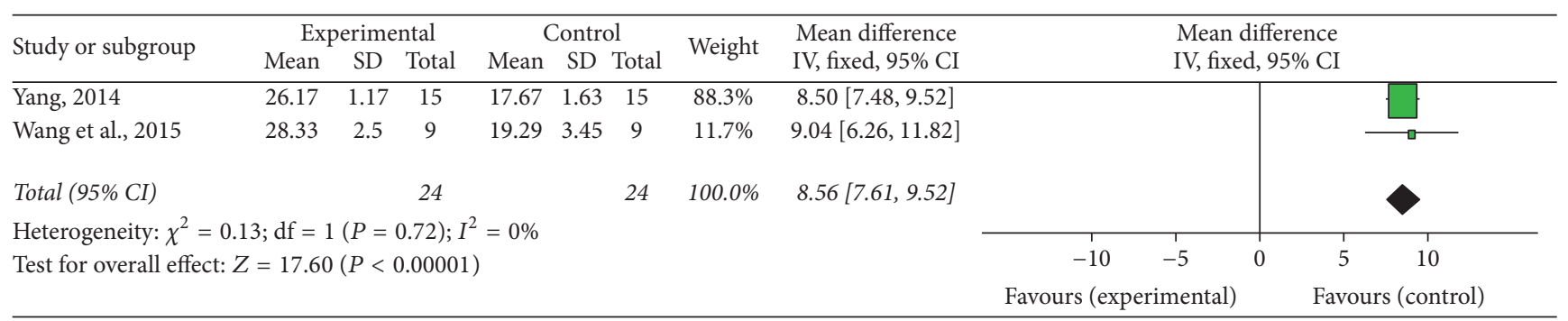

FIGURE 3: The forest plot: effects of G-Rg1 for improving the swim test compared with control group. Note: G-Rg1: Ginsenosides-Rg1.

$34,40]$, antioxidant stress activities in 3 studies $[19,30,36]$, and antiapoptosis in 11 studies $[19,20,22-24,26,30-32$, $35,39]$. The detailed characteristics of included studies are summarized in Table 1.

3.3. Risk of Bias in Included Studies. According to the nineitem modified CAMARADES checklist, the mean quality score of the 25 included studies was 5.12 (interquartile range: 4.75-6.0), with scores ranging from 3 to 7 (Table 2), of which one study [21] got 3 points; five studies [22, 24, 25, 29, 31] got 4 points; eleven studies [19, 20, 23, 26-28, 32-34, 36, 40] got 5 points; six studies [16, 17, 30, 37-39] got 6 points; and two studies $[18,35]$ got 7 points. All studies were published in peer reviewed journals and described random allocation to groups. None of the studies reported blinded assessment of behavioral outcome. Eighteen studies [16-18, 22-26, 28$30,32-35,37-39]$ reported the control of temperature. Six studies $[19,21,22,24,25,29]$ did not use anesthetic without significant intrinsic neuroprotective activity. All of the studies do not have formal sample size calculation. Thirteen studies [17-22, 25, 27, 30, 35-38] reported using of animals without relevant comorbidities (such as aging, diabetes, or hypertension). Three studies [21, 22, 25] did not report the compliance with animal welfare regulations. Seven studies $[16-19,35,39,40]$ stated potential conflict of interests.

\subsection{Effectiveness}

3.4.1. Behavioral Assessments. Fifteen studies, including twelve MPTP-induced PD [16, 17, 22-24, 26, 28, 29, 3234,39 ] and three 6-OHDA induced PD [35-37] studies, used behavioral assessments as primary outcome measures. For the 12 studies on the motor dysfunction of MPTP-induced PD model, 2 studies $[16,17]$ provided clear data of rotarod test,
2 studies [22, 33] provided clear data of swim-score values, 4 studies [16, 17, 23, 39] provided graphical data of pole test, and the other 6 studies [24, 26, 28, 29, 32, 34] were descriptive studies without any data. Meta-analysis of 2 studies [16, 17] reported that the G-Rg1 group significantly improved rotarod test compared with MPTP-injected group ( $n=40$; WMD: 35.75; 95\% CI: 27.20 to 44.31; $P<0.00001$; heterogeneity: $\chi^{2}=0.36 ; \mathrm{df}=1 ; P=0.55 ; I^{2}=0 \%$ ) (Figure 2 ). Meta-analysis of 2 studies $[22,33]$ showed that the G-Rg1 group significantly improved the swim-score values compared with the MPTPinduced PD group ( $n=48$; WMD: 8.56 ; 95\% CI: 7.61 to 9.52 ; $P<0.00001$; heterogeneity: $\chi^{2}=0.13$; df $=1 ; P=0.72$; $I^{2}=0 \%$ ) (Figure 3). Four studies [16, 17, 23, 39] indicated that mice treated with G-Rg1 spent less time descending the pole compared with mice treated with MPTP $(P<0.01$ or $P<0.05$ at different time point). There are two time durations that should be recorded in the pole test: one is the time it took the mouse to turn completely downward (T-turn) and the other one is the time it took the mouse to descend to the floor (T-total). But only one study recorded the two time durations; other studies did not clearly record them in detail. Meanwhile, the climbing pole time of mice in each study was conducted at different days. Owing to the above reasons, meta-analysis for this pole test could not be performed. The other 6 studies $[24,26,28,29,32,34]$ described that GRg1 group significantly improved the motor symptoms of PD induced by MPTP in mice, including the symptoms of thrilling, piloerection, raising tail, activity decrease, postural bradykinesia, and staggering gait but also failed to make a meta-analysis because they were just descriptive studies without any data. For the 3 studies on the motor dysfunction of 6-OHDA induced PD model, 2 studies [35-37] indicated that G-Rg1 group showed significant improvement in the rotational behavior in 6-OHDA-lesioned rats compared with 


\begin{tabular}{|c|c|c|c|c|c|c|}
\hline 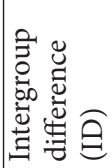 & 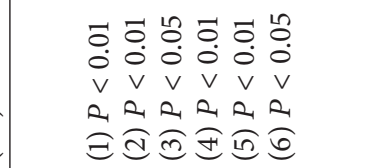 & 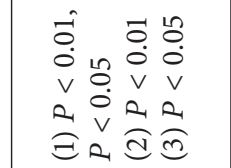 & 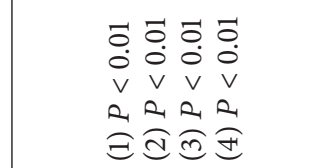 & 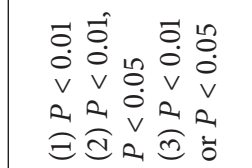 & 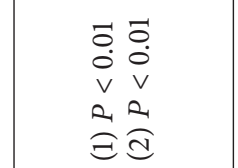 & 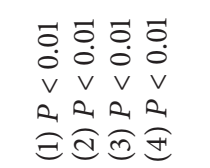 \\
\hline 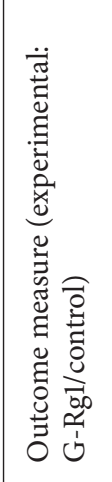 & 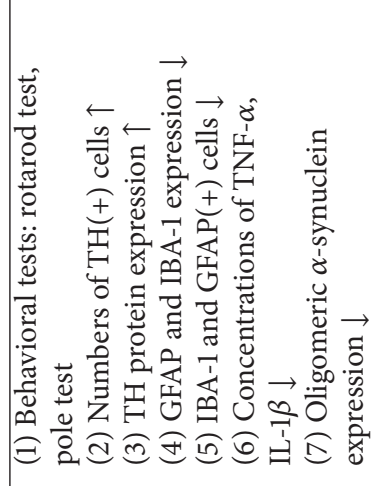 & 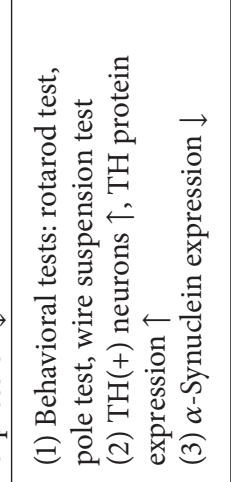 & 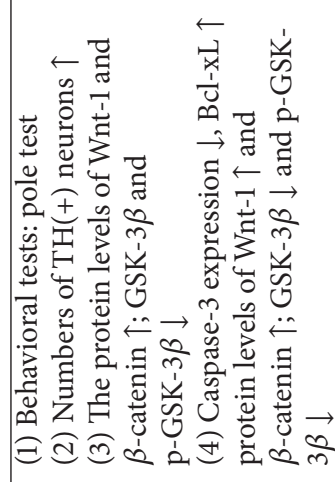 & 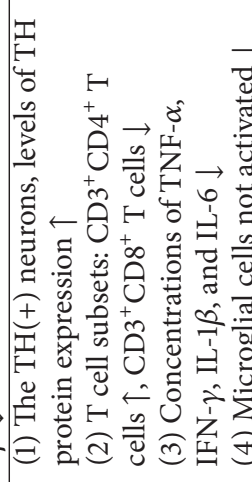 & 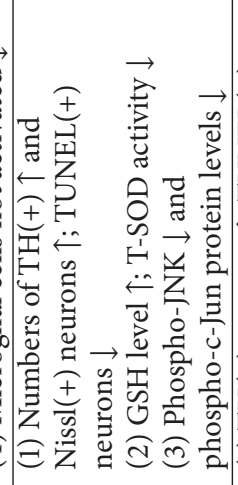 & 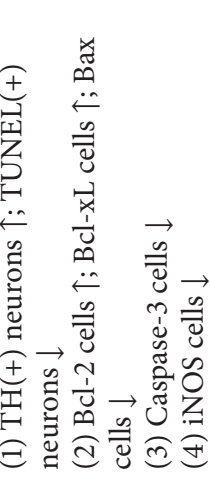 \\
\hline 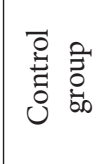 & 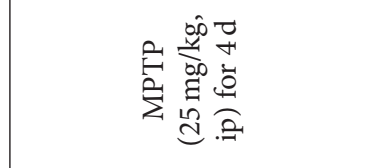 & 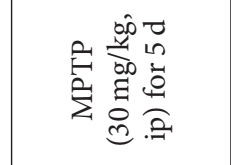 & 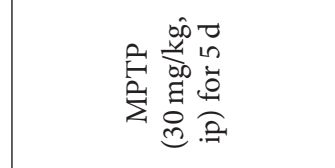 & 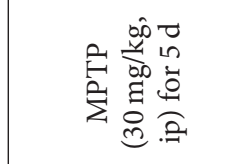 & 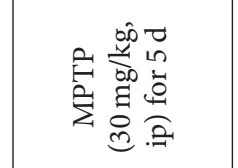 & 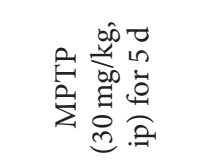 \\
\hline 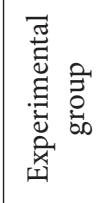 & 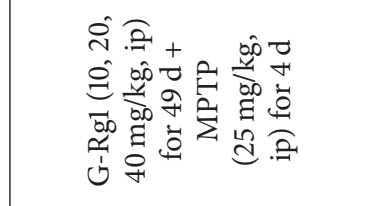 & 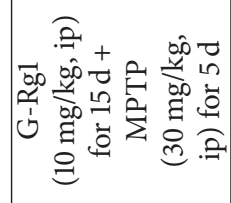 & 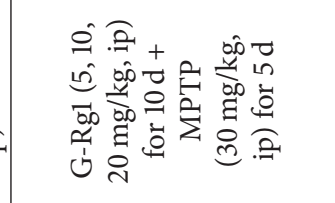 & 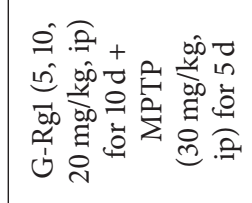 & 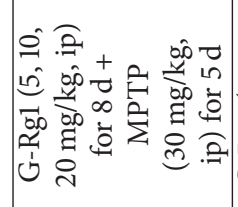 & 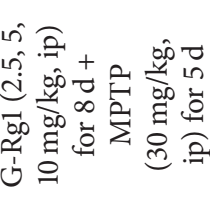 \\
\hline 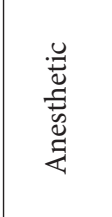 & 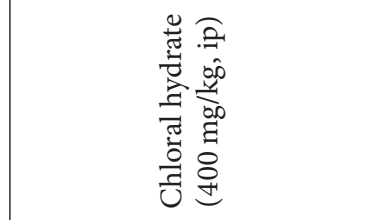 & 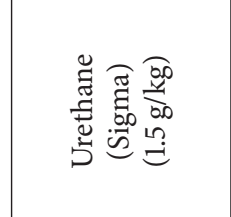 & 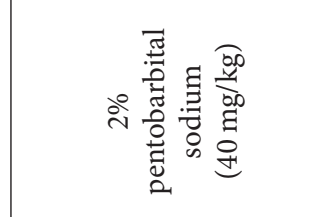 & 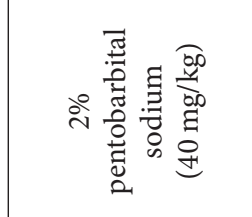 & 孚 & 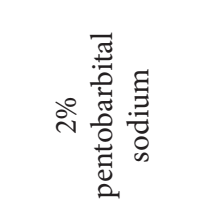 \\
\hline $\begin{array}{l}\overrightarrow{\tilde{v}} \\
\overline{0} \\
\dot{\Xi} \\
\hat{0}\end{array}$ & 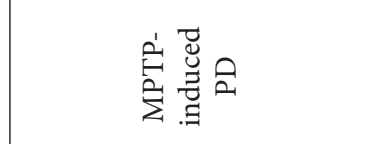 & 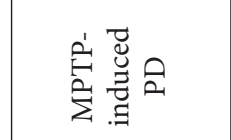 & 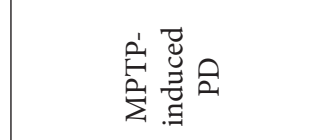 & 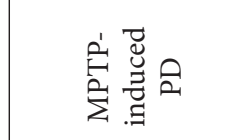 & 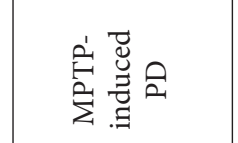 & 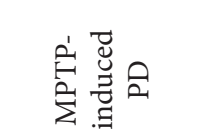 \\
\hline $\begin{array}{l}\frac{1}{50} \\
.00 \\
30 \\
3\end{array}$ & $\begin{array}{l}\infty \\
\stackrel{\infty}{n} \\
\stackrel{\sim}{1} \\
\text { in }\end{array}$ & $\begin{array}{l}\infty \\
0 \\
0 \\
1 \\
\end{array}$ & $\begin{array}{l}\infty \\
\stackrel{\infty}{a} \\
1 \\
\underline{n}\end{array}$ & 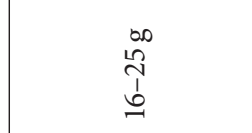 & 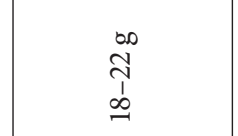 & $\begin{array}{l}\infty \\
1 \\
+1 \\
+1 \\
i\end{array}$ \\
\hline 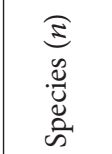 & 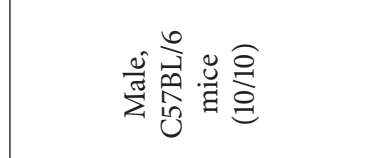 & 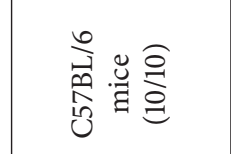 & 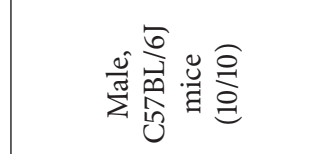 & 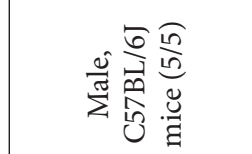 & 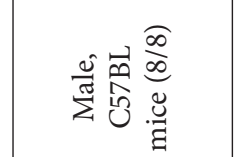 & 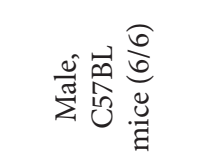 \\
\hline $\begin{array}{l}\text { se } \\
\text { D }\end{array}$ & 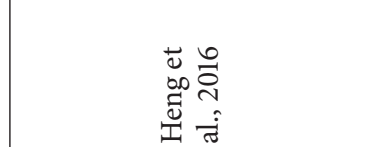 & 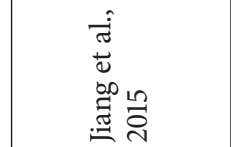 & 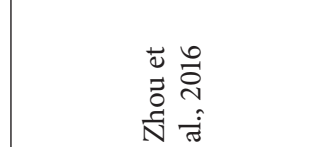 & 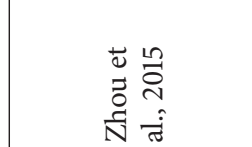 & 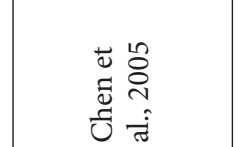 & 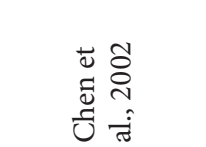 \\
\hline
\end{tabular}




\begin{tabular}{|c|c|c|c|c|c|c|}
\hline 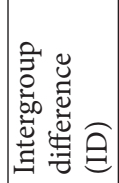 & 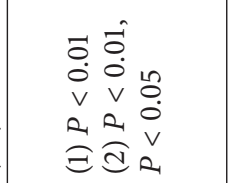 & 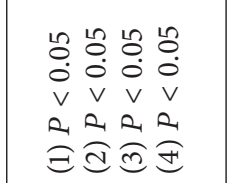 & 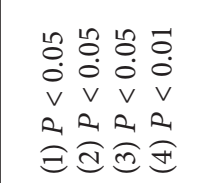 & 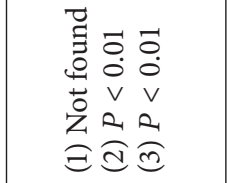 & $\begin{array}{ll}\vec{b} & \overrightarrow{0} \\
0 & 0 \\
v & v \\
0 & 0 \\
2 & a \\
\Xi & \widehat{I}\end{array}$ & 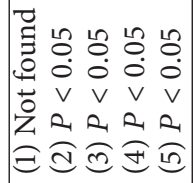 \\
\hline 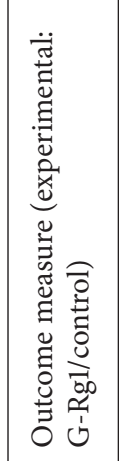 & 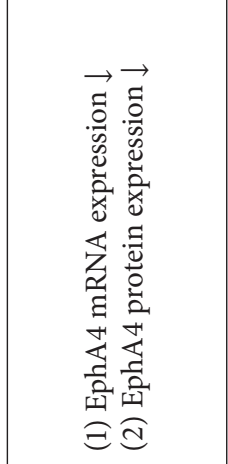 & 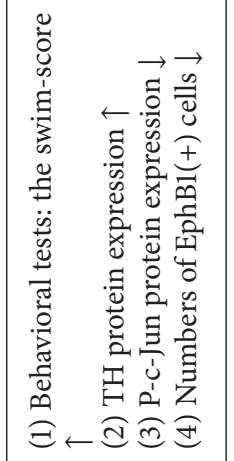 & 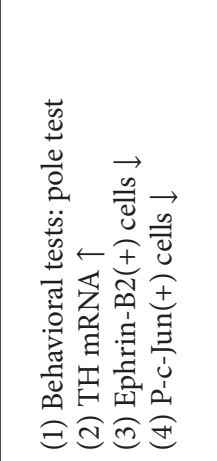 & 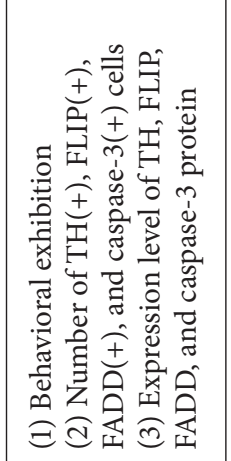 & 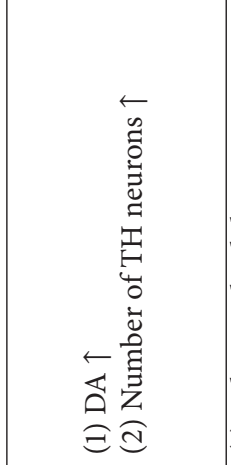 & 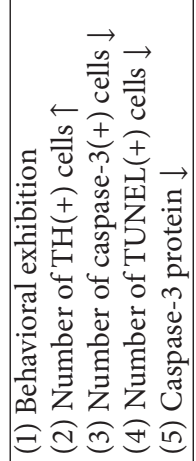 \\
\hline 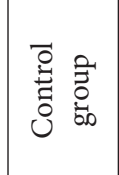 & 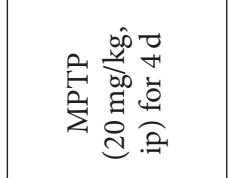 & 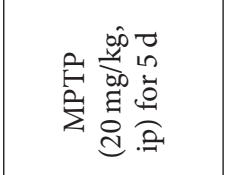 & 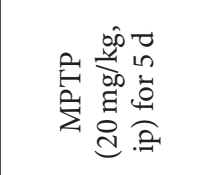 & 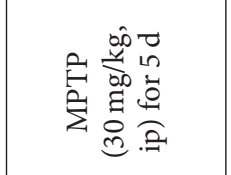 & 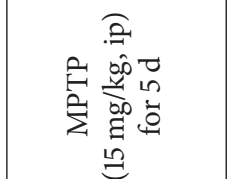 & 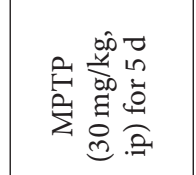 \\
\hline 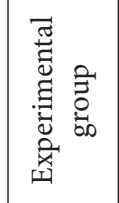 & 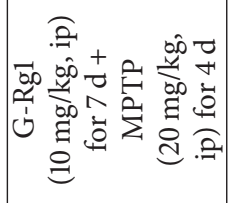 & 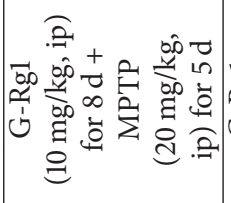 & 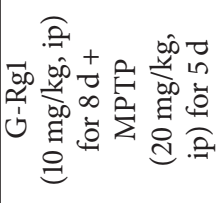 & 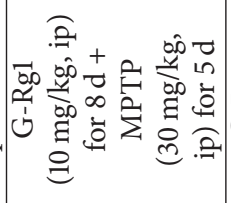 & 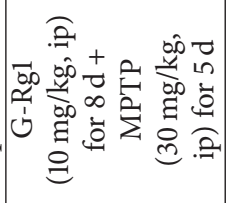 & 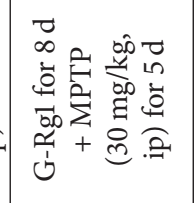 \\
\hline 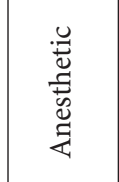 & 岸 & 岸 & 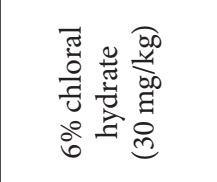 & 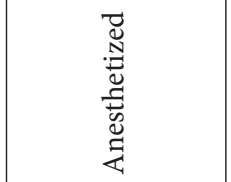 & $\tilde{z}$ & 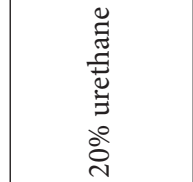 \\
\hline $\begin{array}{l}\overrightarrow{\widetilde{v}} \\
\text { ఏ } \\
\text { a }\end{array}$ & 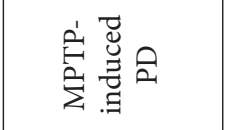 & 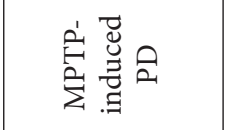 & 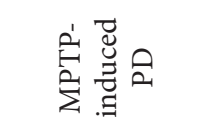 & 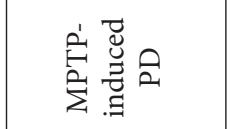 & 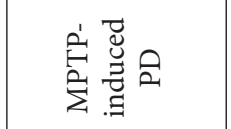 & 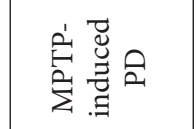 \\
\hline $\begin{array}{l}\frac{7}{60} \\
\frac{00}{20} \\
3\end{array}$ & $\begin{array}{l}\infty \\
0 \\
\stackrel{1}{1} \\
\text { ते }\end{array}$ & $\begin{array}{l}\infty \\
\infty \\
\stackrel{1}{1} \\
\text { ते }\end{array}$ & 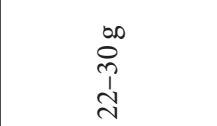 & $\begin{array}{l}\infty \\
0 \\
0 \\
1 \\
\end{array}$ & $\begin{array}{l}\infty \\
\text { n } \\
+1 \\
\stackrel{4}{4}\end{array}$ & $\begin{array}{l}\infty \\
\underset{1}{1} \\
\infty \\
0\end{array}$ \\
\hline $\begin{array}{l}\overrightarrow{\tilde{\Xi}} \\
\text { के }\end{array}$ & 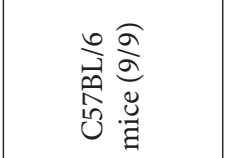 & 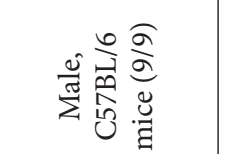 & 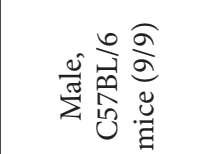 & 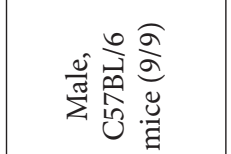 & 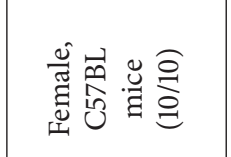 & 茎总 \\
\hline 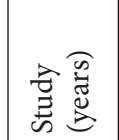 & 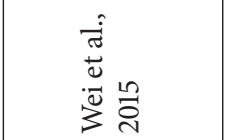 & 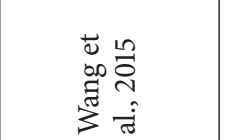 & 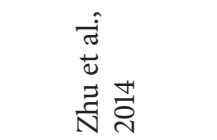 & 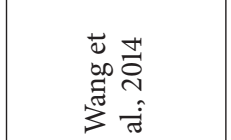 & 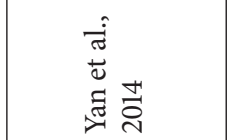 & 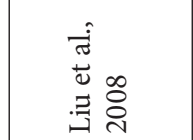 \\
\hline
\end{tabular}




\begin{tabular}{|c|c|c|c|c|c|c|}
\hline 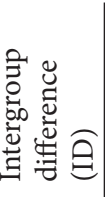 & 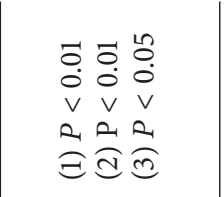 & 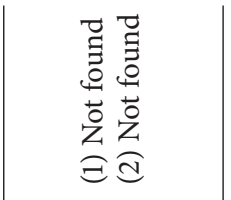 & 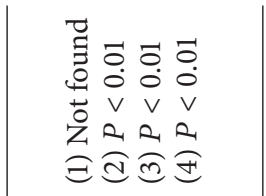 & 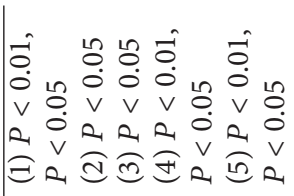 & 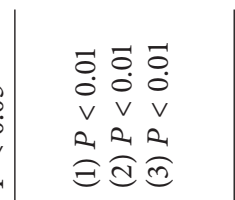 & 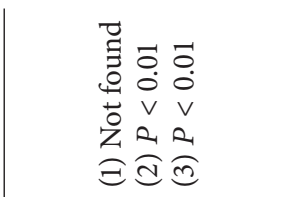 \\
\hline 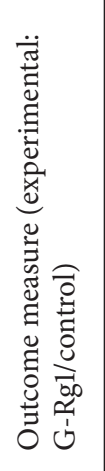 & 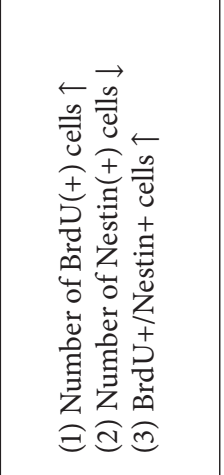 & 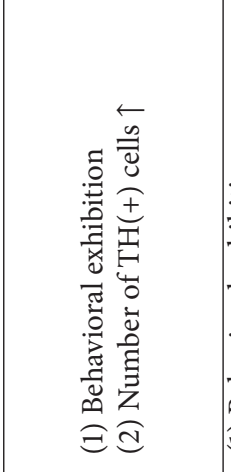 & 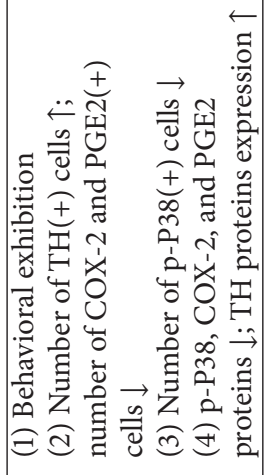 & 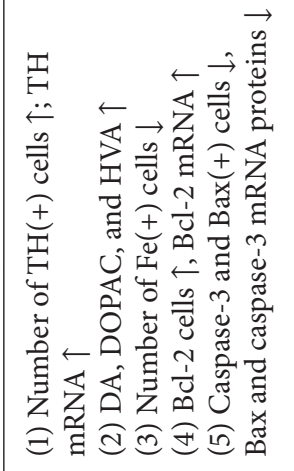 & 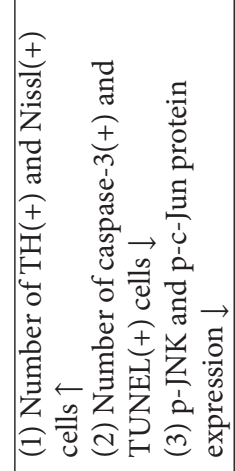 & 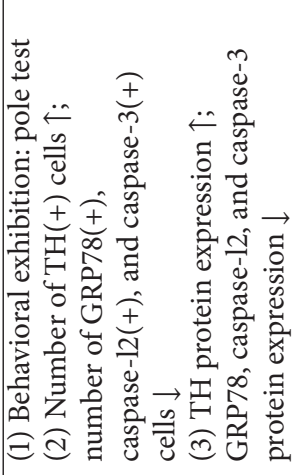 \\
\hline 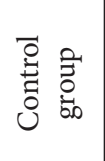 & 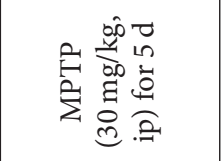 & 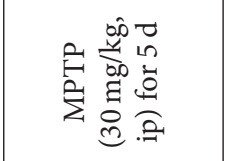 & 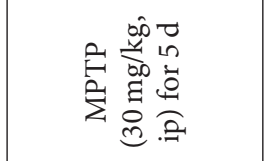 & 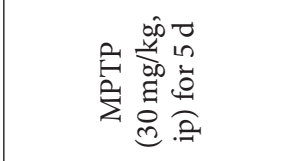 & 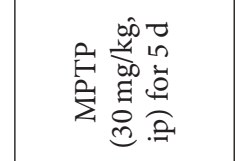 & 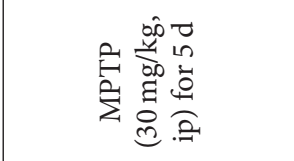 \\
\hline 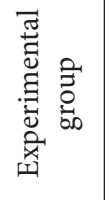 & 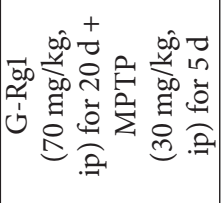 & 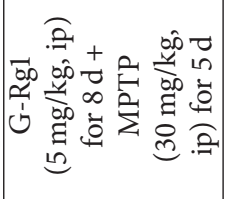 & 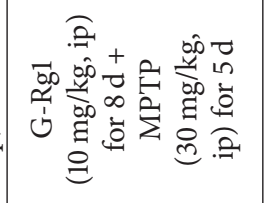 & 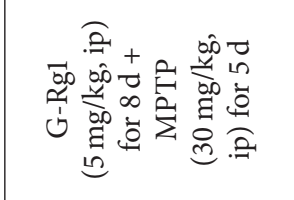 & 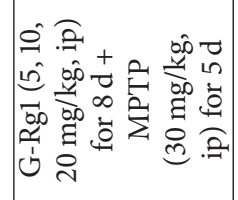 & 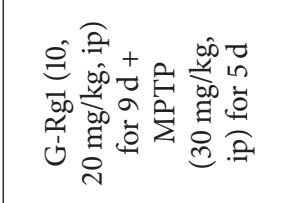 \\
\hline 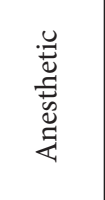 & 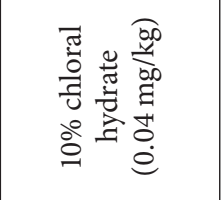 & 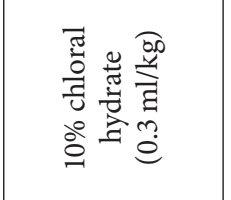 & 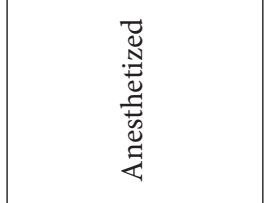 & 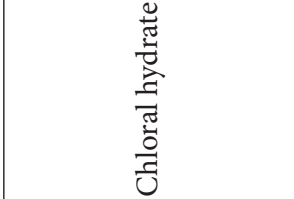 & 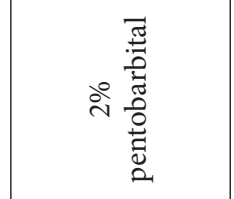 & 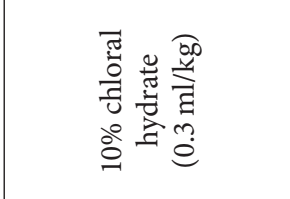 \\
\hline 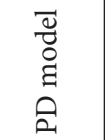 & 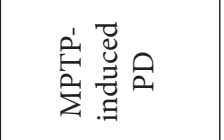 & 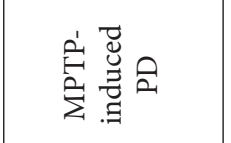 & 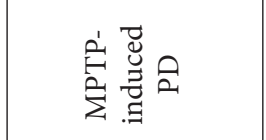 & 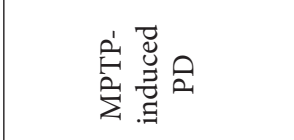 & 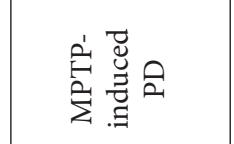 & 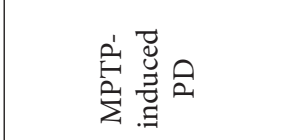 \\
\hline $\begin{array}{l}\frac{7}{3} \\
\frac{0}{800} \\
30 \\
3\end{array}$ & $\begin{array}{l}\infty \\
i n \\
+1 \\
I\end{array}$ & 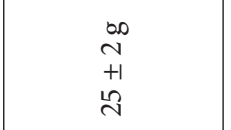 & $\begin{array}{l}\infty \\
\infty \\
\infty \\
\\
\end{array}$ & $\begin{array}{l}\infty \\
\text { in } \\
+1 \\
\stackrel{N}{N}\end{array}$ & $\begin{array}{l}\infty \\
\text { in } \\
+1 \\
\stackrel{1}{1}\end{array}$ & $\begin{array}{l}\infty \\
0 \\
1 \\
1 \\
n\end{array}$ \\
\hline 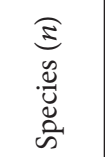 & 竎 & 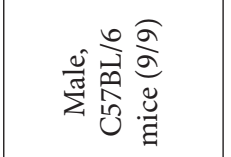 & 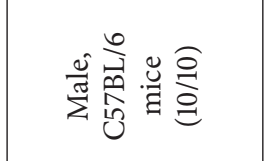 & 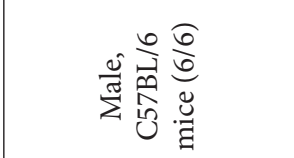 & 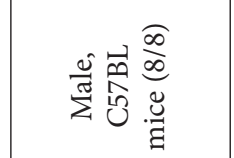 & 伝总 \\
\hline 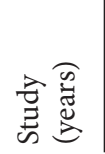 & 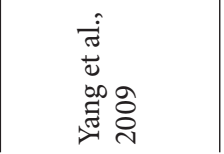 & $\begin{array}{l}\infty \\
\stackrel{0}{0} \\
\text { i } \\
=\end{array}$ & 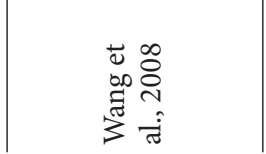 & 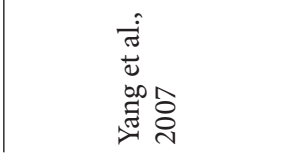 & 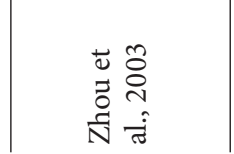 & 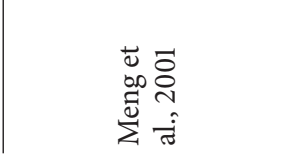 \\
\hline
\end{tabular}




\begin{tabular}{|c|c|c|c|c|c|c|c|}
\hline 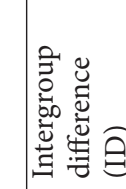 & 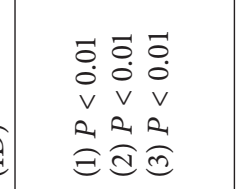 & 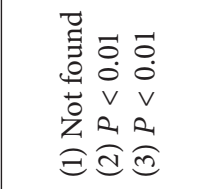 & 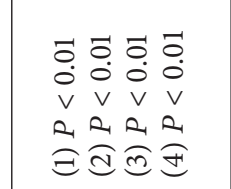 & 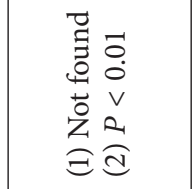 & 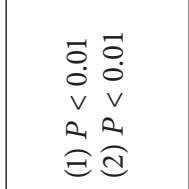 & $\begin{array}{l}\vec{b} \\
\dot{0} \\
v \\
e \\
e \\
\Xi\end{array}$ & 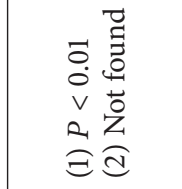 \\
\hline 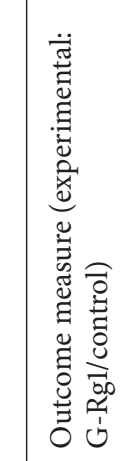 & 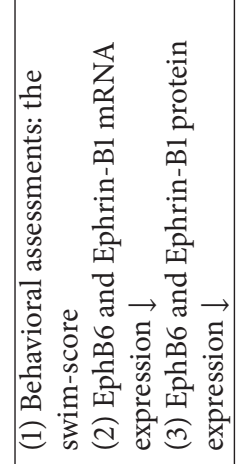 & 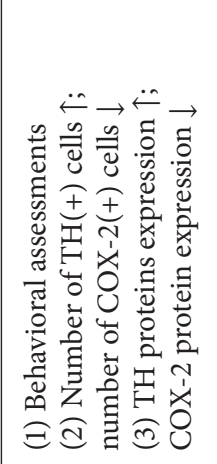 & 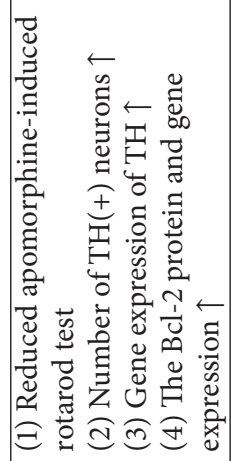 & 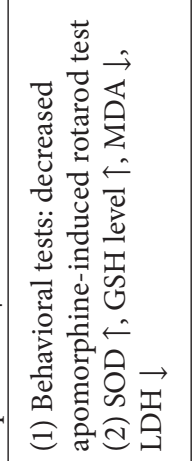 & 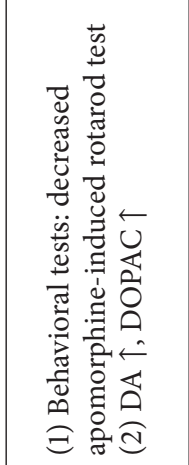 & 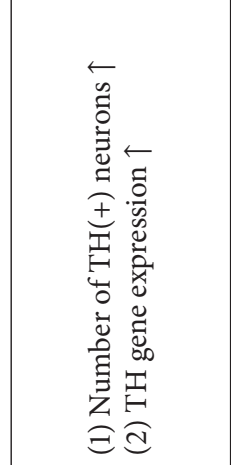 & 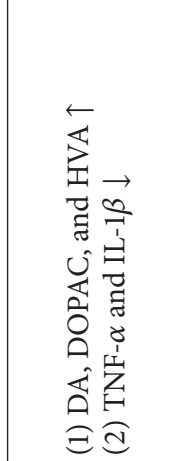 \\
\hline 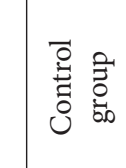 & 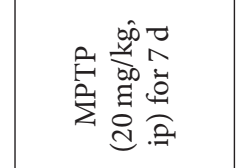 & 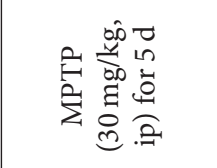 & 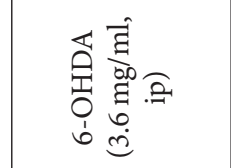 & 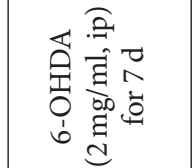 & 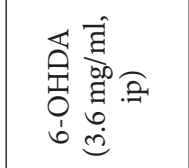 & 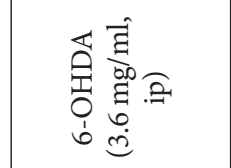 & 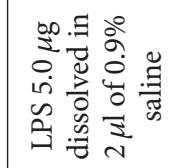 \\
\hline 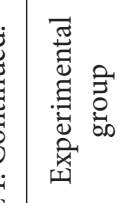 & 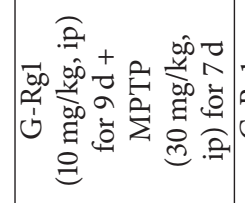 & 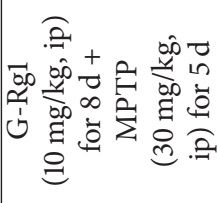 & 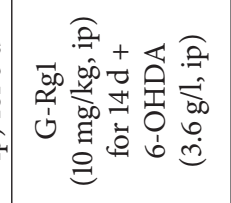 & 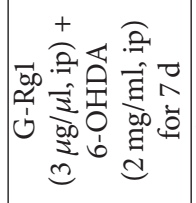 & 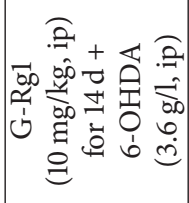 & 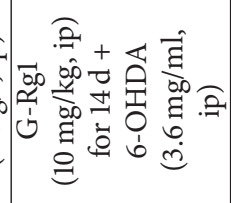 & 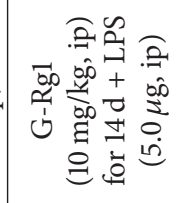 \\
\hline 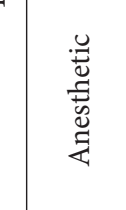 & 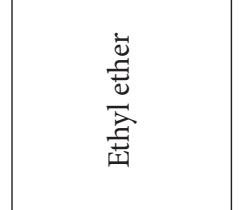 & 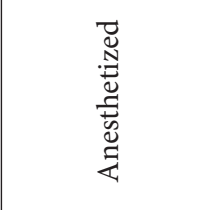 & 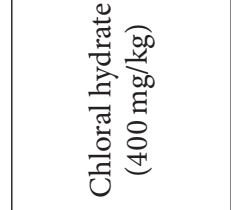 & 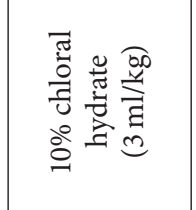 & 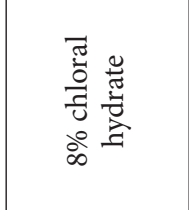 & 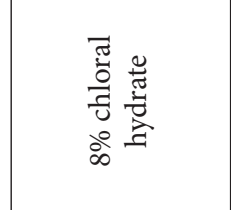 & 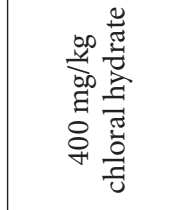 \\
\hline 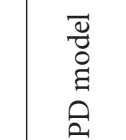 & 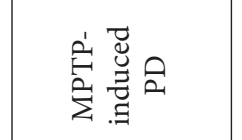 & 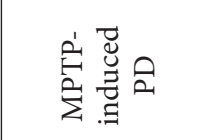 & 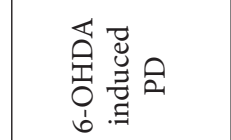 & 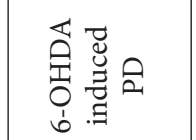 & 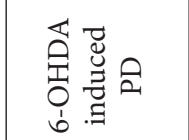 & 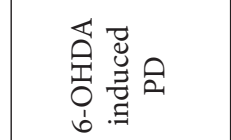 & 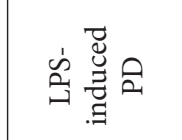 \\
\hline $\begin{array}{l}\frac{7}{600} \\
\frac{.00}{3} \\
3\end{array}$ & 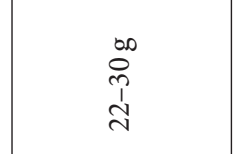 & $\begin{array}{l}\infty \\
0 \\
0 \\
1 \\
\\
\end{array}$ & 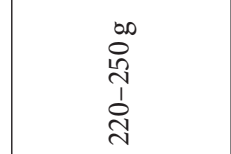 & 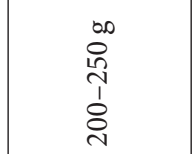 & $\begin{array}{l}\infty \\
0 \\
0 \\
i n \\
1 \\
0 \\
0\end{array}$ & 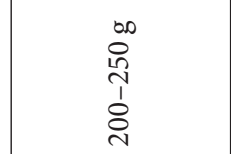 & $\begin{array}{l}\infty \\
0 \\
8 \\
0 \\
1 \\
0 \\
17\end{array}$ \\
\hline 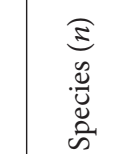 & 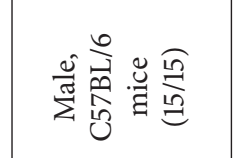 & 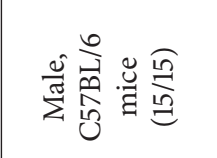 & 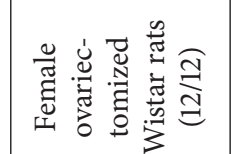 & 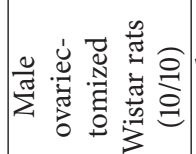 & 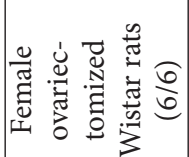 & 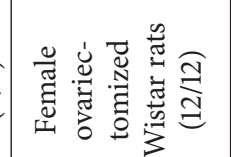 & 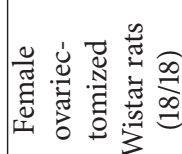 \\
\hline 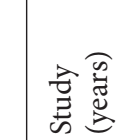 & 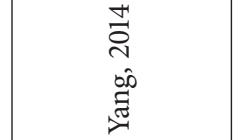 & 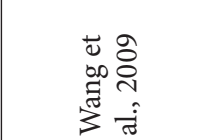 & 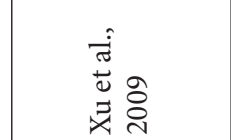 & 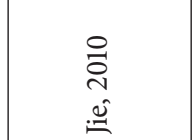 & 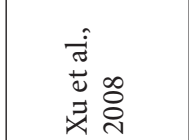 & 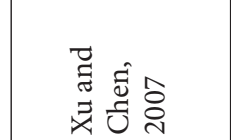 & 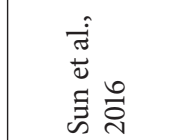 \\
\hline
\end{tabular}


TABLE 2: Quality assessment of included studies.

\begin{tabular}{|c|c|c|c|c|c|c|c|c|c|c|}
\hline Study & $(1)$ & $(2)$ & (3) & $(4)$ & $(5)$ & (6) & $(7)$ & $(8)$ & $(9)$ & Score \\
\hline Heng et al., 2016 & $\sqrt{ }$ & $\sqrt{ }$ & $\sqrt{ }$ & & $\sqrt{ }$ & & & $\sqrt{ }$ & $\sqrt{ }$ & 6 \\
\hline Jiang et al., 2015 & $\sqrt{ }$ & $\sqrt{ }$ & $\sqrt{ }$ & & $\sqrt{ }$ & & $\sqrt{ }$ & $\sqrt{ }$ & $\sqrt{ }$ & 6 \\
\hline Zhou et al., 2015 & $\sqrt{ }$ & $\sqrt{ }$ & $\sqrt{ }$ & & $\sqrt{ }$ & & $\sqrt{ }$ & $\sqrt{ }$ & $\sqrt{ }$ & 7 \\
\hline Chen et al., 2005 & $\sqrt{ }$ & & $\sqrt{ }$ & & & & $\sqrt{ }$ & $\sqrt{ }$ & $\sqrt{ }$ & 5 \\
\hline Chen et al., 2002 & $\sqrt{ }$ & & $\sqrt{ }$ & & $\sqrt{ }$ & & $\sqrt{ }$ & $\sqrt{ }$ & & 5 \\
\hline Wei et al., 2015 & $\sqrt{ }$ & & $\sqrt{ }$ & & & & $\sqrt{ }$ & & & 3 \\
\hline Wang et al., 2015 & $\sqrt{ }$ & $\sqrt{ }$ & $\sqrt{ }$ & & & & $\sqrt{ }$ & & & 4 \\
\hline Zhu et al., 2014 & $\sqrt{ }$ & $\sqrt{ }$ & $\sqrt{ }$ & & $\sqrt{ }$ & & & $\sqrt{ }$ & & 5 \\
\hline Wang et al., 2014 & $\sqrt{ }$ & $\sqrt{ }$ & $\sqrt{ }$ & & & & & $\sqrt{ }$ & & 4 \\
\hline Yan et al., 2014 & $\sqrt{ }$ & $\sqrt{ }$ & $\sqrt{ }$ & & & & $\sqrt{ }$ & & & 4 \\
\hline Liu et al., 2008 & $\sqrt{ }$ & $\sqrt{ }$ & $\sqrt{ }$ & & $\sqrt{ }$ & & & $\sqrt{ }$ & & 5 \\
\hline Yang et al., 2009 & $\sqrt{ }$ & & $\sqrt{ }$ & & $\sqrt{ }$ & & $\sqrt{ }$ & $\sqrt{ }$ & & 5 \\
\hline Ji, 2008 & $\sqrt{ }$ & $\sqrt{ }$ & $\sqrt{ }$ & & $\sqrt{ }$ & & & $\sqrt{ }$ & & 5 \\
\hline Wang et al., 2008 & $\sqrt{ }$ & $\sqrt{ }$ & $\sqrt{ }$ & & & & & $\sqrt{ }$ & & 4 \\
\hline Yang et al., 2007 & $\sqrt{ }$ & $\sqrt{ }$ & $\sqrt{ }$ & & $\sqrt{ }$ & & $\sqrt{ }$ & $\sqrt{ }$ & & 6 \\
\hline Zhou et al., 2003 & $\sqrt{ }$ & & $\sqrt{ }$ & & $\sqrt{ }$ & & & $\sqrt{ }$ & & 4 \\
\hline Meng et al., 2001 & $\sqrt{ }$ & $\sqrt{ }$ & $\sqrt{ }$ & & $\sqrt{ }$ & & & $\sqrt{ }$ & & 5 \\
\hline Yang, 2014 & $\sqrt{ }$ & $\sqrt{ }$ & $\sqrt{ }$ & & $\sqrt{ }$ & & & $\sqrt{ }$ & & 5 \\
\hline Wang et al., 2009 & $\sqrt{ }$ & $\sqrt{ }$ & $\sqrt{ }$ & & $\sqrt{ }$ & & & $\sqrt{ }$ & & 5 \\
\hline Xu et al., 2009 & $\sqrt{ }$ & $\sqrt{ }$ & $\sqrt{ }$ & & $\sqrt{ }$ & & $\sqrt{ }$ & $\sqrt{ }$ & $\sqrt{ }$ & 7 \\
\hline Jie, 2010 & $\sqrt{ }$ & & $\sqrt{ }$ & & $\sqrt{ }$ & & $\sqrt{ }$ & $\sqrt{ }$ & & 5 \\
\hline Xu et al., 2008 & $\sqrt{ }$ & $\sqrt{ }$ & $\sqrt{ }$ & & $\sqrt{ }$ & & $\sqrt{ }$ & $\sqrt{ }$ & & 6 \\
\hline $\mathrm{Xu}$ and Chen, 2007 & $\sqrt{ }$ & $\sqrt{ }$ & $\sqrt{ }$ & & $\sqrt{ }$ & & $\sqrt{ }$ & $\sqrt{ }$ & & 6 \\
\hline Zhou et al., 2016 & $\sqrt{ }$ & $\sqrt{ }$ & $\sqrt{ }$ & & $\sqrt{ }$ & & $\sqrt{ }$ & $\sqrt{ }$ & & 6 \\
\hline Sun et al., 2016 & $\sqrt{ }$ & & $\sqrt{ }$ & & $\sqrt{ }$ & & $\sqrt{ }$ & $\sqrt{ }$ & & 5 \\
\hline
\end{tabular}

Note: (1) peer reviewed publication; (2) control of temperature; (3) random allocation to groups; (4) blinded assessment of behavioral outcome; (5) use of anesthetic without significant intrinsic neuroprotective activity; (6) calculation of the sample size necessary to achieve sufficient power; (7) appropriate animal model (aged, diabetic, or hypertensive); (8) compliance with animal welfare regulations; (9) statement of potential conflict of interests.

control group; one study [17] reported that, in comparison with G-Rg1, mice treated with MPTP spent much more time reaching the platform during the wire suspension test $(P<$ 0.05).

3.4.2. The Number of TH-Positive Dopamine Neurons. Sixteen studies, including fourteen MPTP-induced PD [16-20, 24, 26, 28-32, 34, 39] and two 6-OHDA induced PD [35, 38] studies, demonstrated the number of TH-positive dopamine neurons in the SNpc by immunohistochemistry analysis. Eleven out of 14 MPTP-induced PD studies provided raw data to make meta-analysis. Meta-analysis of 11 studies showed that G-Rg1 significantly improved the number of TH-positive neurons when compared with that in the MPTP-induced group $(n=$ 180; WMD: 36.78; 95\% CI: 35.27 to $38.28 ; P<0.00001$; heterogeneity: $\chi^{2}=368.15 ; \mathrm{df}=10 ; P<0.00001 ; I^{2}=$ $97 \%)$. Meanwhile, there was obvious heterogeneity for the analysis of TH-positive neurons between studies. Several factors were found to make significant influence on the outcome measure. When the authors counted the number of TH-positive neurons, the different types of microscopes, various magnification (such as $\times 10, \times 40, \times 100$, and $\times 200$ ), different sample drawing areas of the substantia nigra, different numbers of specimens of brain glass (such as 3 brain slices or
5 brain slices), different slices of brain tissue thickness (such as cut into $20 \mu \mathrm{m}$ and $30 \mu \mathrm{m}$ ), and use of diverse anesthetics (such as chloral hydrate, pentobarbital sodium, and urethane) in different studies may contribute to this discrepancy. Thus, those reasons were considered as the potential sources of the heterogeneity. Seven studies [16-18, 22, 24, 32, 34] which reported the level of TH protein expression were qualified to perform a meta-analysis, and the random-effect model was applied for statistical analysis account for the heterogeneity ( $n=82$; SMD: 5.56 ; 95\% CI: 3.56 to $7.56 ; P<0.00001$; heterogeneity: $\chi^{2}=18.24$; $\mathrm{df}=6 ; P=0.006 ; I^{2}=67 \%$ ) favouring G-Rg1 when compared with controls. We used sensitivity analyses omitting each study at a time from the original analysis. After removing 1 study [22] which was considered to be the potential source of the heterogeneity, the remaining 6 studies reported that G-Rgl significantly improved the level of TH protein expression compared with control group ( $n=64$; SMD: 4.46 ; $95 \%$ CI: 3.15 to 5.76 ; $P<0.00001$; heterogeneity: $\chi^{2}=6.54$; df $=5 ; I^{2}=$ $23 \%$ ) (Figure 4). Two studies [23, 30] showed that G-Rg1 significantly increased the expression of TH mRNA in the substantia nigra of mice compared with the control group ( $n=30$; WMD: 2.07 ; 95\% CI: 1.13 to $3.01 ; P<0.00001$; heterogeneity: $\chi^{2}=0.01 ; \mathrm{df}=1 ; P=0.93 ; I^{2}=0 \%$ ) (Figure 5). 


\begin{tabular}{|c|c|c|c|c|c|c|c|c|c|c|c|c|c|}
\hline \multirow{2}{*}{ Study or subgroup } & \multicolumn{3}{|c|}{ Experimental } & \multicolumn{3}{|c|}{ Control } & \multirow{2}{*}{ Weight } & \multirow{2}{*}{$\begin{array}{l}\text { Std. mean difference } \\
\text { IV, random, } 95 \% \text { CI }\end{array}$} & \multirow{2}{*}{\multicolumn{5}{|c|}{$\begin{array}{l}\text { Std. mean difference } \\
\text { IV, random, } 95 \% \text { CI }\end{array}$}} \\
\hline & Mean & SD & Total & Mean & SD & Total & & & & & & & \\
\hline Heng et al., 2016 & 0.65 & 0.06 & 4 & 0.36 & 0.06 & 4 & $13.4 \%$ & $4.20[1.00,7.41]$ & \multicolumn{5}{|c|}{0} \\
\hline Jiang et al., 2015 & 99.01 & 10.73 & 5 & 52.07 & 14.2 & 5 & $22.6 \%$ & $3.37[1.10,5.63]$ & \multicolumn{5}{|c|}{ 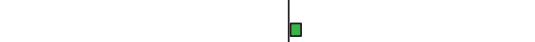 } \\
\hline Meng et al., 2001 & 0.77 & 0.066 & 6 & 0.54 & 0.05 & 6 & $25.0 \%$ & $3.63[1.53,5.73]$ & \multicolumn{5}{|c|}{$\square$} \\
\hline Wang et al., 2009 & 9.5 & 0.8 & 6 & 5.4 & 0.5 & 6 & $15.0 \%$ & $5.67[2.68,8.67]$ & \multicolumn{5}{|c|}{$\square$} \\
\hline Wang et al., 2014 & 26.92 & 1.34 & 6 & 12.33 & 1.45 & 6 & $6.6 \%$ & $9.65[4.80,14.49]$ & \multicolumn{5}{|c|}{$\square$} \\
\hline Zhou et al., 2015 & 0.93 & 0.06 & 5 & 0.69 & 0.04 & 5 & $17.6 \%$ & $4.25[1.56,6.95]$ & \multicolumn{5}{|c|}{$\square$} \\
\hline \multicolumn{3}{|l|}{ Total (95\% CI) } & \multicolumn{3}{|l|}{32} & 32 & $100.0 \%$ & $4.46[3.15,5.76]$ & & & \multicolumn{3}{|l|}{1} \\
\hline \multirow{2}{*}{\multicolumn{9}{|c|}{$\begin{array}{l}\text { Heterogeneity: } \tau^{2}=0.62 ; \chi^{2}=6.54 ; \mathrm{df}=5(P=0.26) ; I^{2}=23 \% \\
\text { Test for overall effect: } Z=6.71(P<0.00001)\end{array}$}} & & & & & \\
\hline & & & & & & & & & -100 & -50 & 0 & 50 & 100 \\
\hline & & & & & & & & & \multicolumn{3}{|c|}{ Favours (experimental) } & \multicolumn{2}{|c|}{ Favours (control) } \\
\hline
\end{tabular}

Figure 4: The forest plot: effects of G-Rg1 for improving the level of TH protein expression compared with control group. Note: G-Rg1: Ginsenosides-Rg1; TH: Tyrosine Hydroxylase.

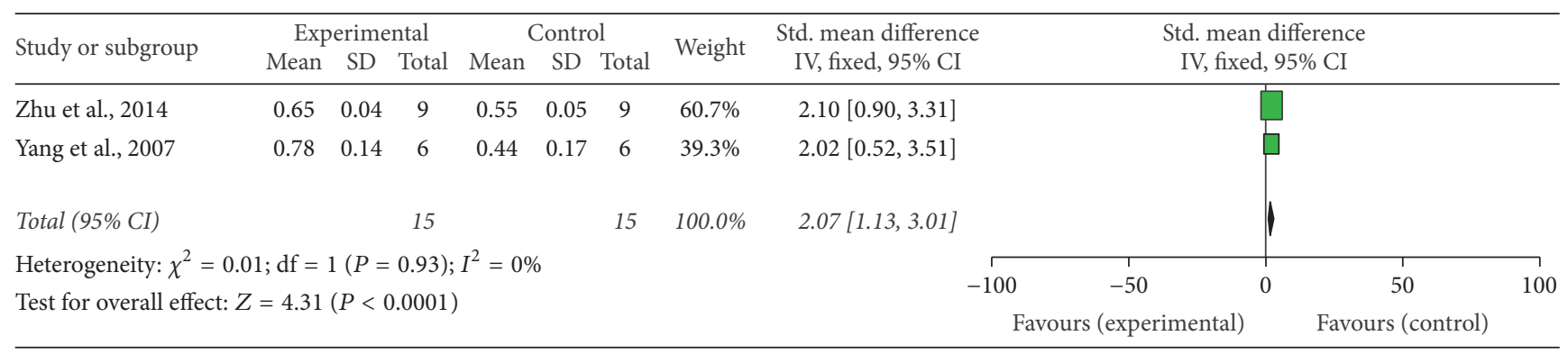

Figure 5: The forest plot: effects of G-Rg1 for improving number of TH mRNA compared with control group. Note: G-Rg1: Ginsenosides-Rg1; TH: Tyrosine Hydroxylase.

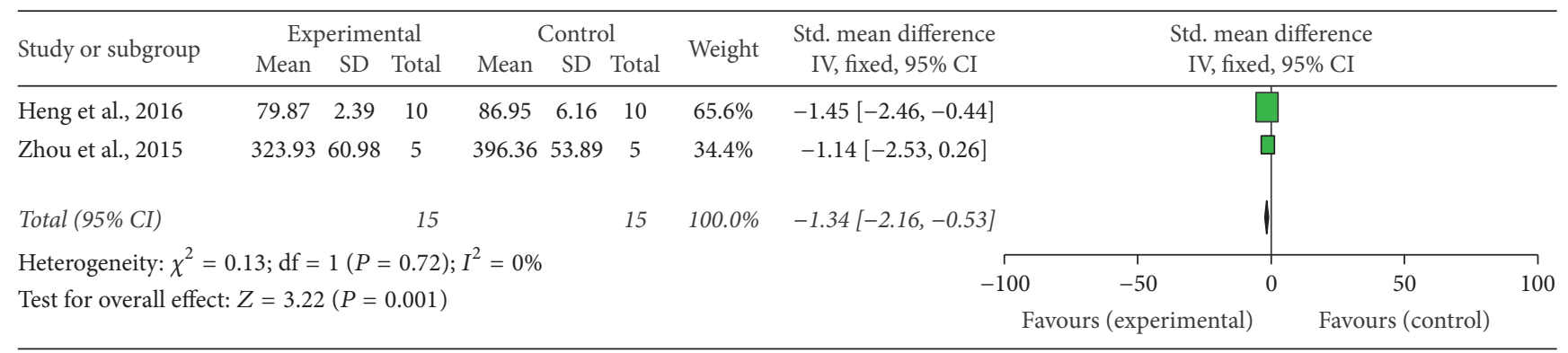

FIGURE 6: The forest plot: effects of G-Rg1 for decreasing the concentrations of IL-1 $\beta$ compared with control group. Note: G-Rg1: GinsenosidesRg1; IL-1 $\beta$ : cytokine interleukin-1 $\beta$.

\subsubsection{The Mechanisms of Neuroprotective Function of G-Rg1 in PD}

Anti-Inflammatory Activities. Ten studies [16-18, 21-23, 29, $33,34,40]$ reported the anti-inflammatory effect of G-Rg1 on the PD mice induced by MPTP $(n=7)$, 6-OHDA $(n=2)$, and LPS $(n=1)$ in the SNpc. Among them, only 2 studies $[16,18]$ reported the change of concentrations of cytokine interleukin- $1 \beta$ (IL- $1 \beta$ ), whereas the other 8 studies failed to be pooled for analysis due to use of different anti-inflammatory indicators once or the absence of data. Meta-analysis of 2 studies $[16,18]$ showed that the concentrations of cytokine interleukin-1 $\beta$ (IL-1 $\beta$ ) in the G-Rgl groups significantly decreased compared with the control group ( $n=40$; SMD: -1.32 ; 95\% CI: -2.02 to $-0.62 ; P=0.0002$; heterogeneity: $\chi^{2}=0.12 ; \mathrm{df}=1 ; P=0.73 ; I^{2}=0 \%$ ) (Figure 6). Three studies $[16,18,40]$ also showed that tumor necrosis factor- $\alpha$ (TNF- $\alpha$ ), interferon- $\gamma$ (IFN- $\gamma$ ), and IL-6 in the G-Rg1 groups significantly decreased compared with the control group $(P<$ 0.01 or $P<0.05)$. Three studies [21, 22, 33] reported significant effects of G-Rg1 for decreasing the expression of erythropoietin-producing hepatocellular cell line such as EphA4, EphB6, and EphB1 compared with the control group in the SNpc $(P<0.01$ or $P<0.05)$. One study [16] showed that G-Rg1 groups significantly decreased the expression of IBA-1 and GFAP proteins and the number of IBA-1and GFAP-positive cells compared with the control group 


\begin{tabular}{|c|c|c|c|c|c|c|c|c|c|c|c|c|}
\hline \multirow{3}{*}{$\begin{array}{l}\text { Study or subgroup } \\
\text { Chen et al., } 2005\end{array}$} & \multicolumn{3}{|c|}{ Experimental } & \multicolumn{3}{|c|}{ Control } & \multirow{2}{*}{ Weight } & \multirow{2}{*}{$\begin{array}{l}\text { Mean difference } \\
\text { IV, fixed, } 95 \% \text { CI }\end{array}$} & \multirow{2}{*}{\multicolumn{4}{|c|}{$\begin{array}{l}\text { Mean difference } \\
\text { IV, fixed, } 95 \% \text { CI }\end{array}$}} \\
\hline & Mean & SD & Total & Mean & SD & Total & & & & & & \\
\hline & 15.41 & 1.81 & 8 & 25.18 & 1.27 & 8 & $31.1 \%$ & $-9.77[-11.30,-8.24]$ & $\rightarrow \square$ & & & \\
\hline Chen et al., 2002 & 14.3 & 1 & 6 & 23.76 & 1.88 & 6 & $25.1 \%$ & $-9.46[-11.16,-7.76]$ & $\rightarrow-$ & & & \\
\hline Liu et al., 2008 & 11.34 & 3.45 & 15 & 20.63 & 2.82 & 15 & $14.3 \%$ & $-9.29[-11.54,-7.04]$ & $\longrightarrow$ & & & \\
\hline Zhou et al., 2003 & 15.38 & 1.66 & 8 & 25.09 & 1.55 & 8 & $29.5 \%$ & $-9.71[-11.28,-8.14]$ & $-\square$ & & & \\
\hline Total (95\% CI) & & & 37 & & & 37 & $100.0 \%$ & $-9.61[-10.46,-8.75]$ & 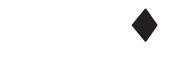 & & & \\
\hline \multirow{2}{*}{\multicolumn{9}{|c|}{$\begin{array}{l}\text { Heterogeneity: } \chi^{2}=0.16 ; \mathrm{df}=3(P=0.98) ; I^{2}=0 \% \\
\text { Test for overall effect: } Z=22.04(P<0.00001)\end{array}$}} & -10 & -5 & 5 & 10 \\
\hline & & & & & & & & & Favours (experir & mental) & Favol & rs (control) \\
\hline
\end{tabular}

FIGURE 7: The forest plot: effects of G-Rg1 for improving TUNEL expression compared with control group. Note: G-Rg1: Ginsenosides-Rg1.

$(P<0.01)$. Two studies $[29,34]$ showed that G-Rg1 reduced COX-2 expression in the SN and might act on the P38 signaling pathway to protect the DA neurons in PD $(P<$ $0.01)$.

Antioxidant Stress. Three studies including two MPTPinduced PD $[19,30,36]$ and one 6-OHDA induced PD [36] studies reported the antioxidant stress effect of G-Rg1 on PD models. Two studies $[19,36]$ detected that G-Rg1 significantly increased glutathione (GSH) level and decreased total superoxide dismutase (T-SOD) activity and lactate dehydrogenase (LDH) levels in the SN compared with the control group $(P<0.01)$. The remaining study [30] showed significant effects of G-Rgl for reducing the numbers of ironstaining cells compared with the control group $(P<0.01)$.

Antiapoptosis. Eleven studies [19, 20, 22-24, 26, 30-32, 35, 39] reported the effect of G-Rg1 against MPTP-induced $(n=10)$ or 6-OHDA $(n=1)$ induced apoptosis in mouse SN neurons. Four studies $[19,20,26,31]$ used the number of TUNELpositive neurons as one of the indicators of antiapoptotic activities mechanisms. Meta-analysis of 4 studies [19, 20, $26,31]$ showed that pretreatment with G-Rg1 remarkably decreased the TUNEL-positive neurons in the SN compared with the control $(n=74$; WMD: -9.61 ; $95 \%$ CI: -10.46 to $-8.75 ; P<0.00001$; heterogeneity: $\chi^{2}=0.16 ; \mathrm{df}=3 ; P=0.98$; $I^{2}=0 \%$ ) (Figure 7). The other 7 studies failed to be pooled for analysis due to lack of the data of TUNEL-positive neurons. Three studies [20, 30, 35] showed that G-Rg1 significantly increased the number of Bcl-2 and Bcl-xL cells compared with the control $(P<0.01$ or $P<0.05)$. Six studies $[20,24$, $26,30-32,39]$ reported that G-Rg1 remarkably decreased the number of caspase- 3 positive cells in the $\mathrm{SN}$ compared with the control $(P<0.01$ or $P<0.05)$. Three studies [22, 23, 31] reported that G-Rg1 dramatically decreased phospho-JNK and phospho-c-Jun protein expression compared with the control $(P<0.01$ or $P<0.05)$.

\section{Discussion}

4.1. Summary of Evidence. Twenty-five studies with 516 animals were identified. This study found that G-Rgl could improve the neurobehavioral abnormality and exert potential neuroprotective effects on PD model through different mechanisms such as antineuroinflammation, antioxidant stress, and antiapoptosis. However, we should treat the preclinical evidences cautiously because the methodological flaws undermine the validity of outcomes.

4.2. Methodological Considerations. This systematic review has a number of weaknesses. Firstly, animal studies with neutral or negative results may be more likely to remain unpublished and will be missed. Therefore, the effect size may be overstated. Secondly, our search strategy includes only Chinese or English databases, which may cause a certain degree of selective bias [41]. Thirdly, previous meta-analyses have suggested that animal studies that are less rigorously designed may overestimate treatment effects [42]. In the present study, all the studies failed to mention the blinded assessment of behavioral outcome. It may lead to performance bias and detection bias [43]. Sufficient size is essential to determine the efficacy of a new therapy or drug [44]. No study reported the calculation of the sample size that was necessary to achieve sufficient power, which indicated the lack of statistical power to ensure suitable estimation of the therapeutic effect [45]. Finally, the results from individual studies were inconsistent, and most of the studies used the graph rather than original data to present the outcomes. Therefore, we could not synthesize these data into the quantity.

4.3. Possible Neuroprotective Mechanism. The possible mechanisms of neuroprotective activity of G-Rg1 in PD are summarized as follows. (i) Inhibiting oxidative stress: high reactive iron levels can yield excess hydrogen peroxide and other reactive oxygen species (ROS), which will lead to mitochondrial dysfunction and increased dopamine metabolism. G-Rg1 could reduce the number of iron-staining cells in the SN of MPTP treated mouse [30] and showed protective effect. As one of the most important antioxidant molecules, GSH could clear $\mathrm{H}_{2} \mathrm{O}_{2}$ and prevent its reaction with iron to form the highly reactive ${ }^{\circ} \mathrm{OH}$ radical in the Fenton reaction. The present study showed that pretreatment with G-Rg1 could protect antioxidant defense system through attenuating the loss of GSH and increasing activity of TSOD (including $\mathrm{Cu} / \mathrm{Zn}-\mathrm{SOD}$ and $\mathrm{Mn}$-SOD) following MPTP treatment [19]. (ii) Inhibiting neuroinflammation: animal, 
human, epidemiologic, and therapeutic studies all revealed that the neuroinflammatory cascade plays a key role in the pathogenesis of PD. Recent studies demonstrated that G-Rg1 notably decreased neuroinflammation levels in the SNpc induced by MPTP. G-Rg1 could decrease the level of IBA-1, GFAP, EhpA, and EhpB protein expression, IBA1, GFAP, EhpA, and EhpB positive cells, phosphorylated p38, COX-2, and PGE2 proteins, TNF- $\alpha$, IL- $1 \beta$, and the oligomeric $\alpha$-synuclein expression in the SNpc [16-18, 21, 22]. (iii) Decreasing toxin-induced apoptosis: the protective effect of G-Rg1 against neurons apoptosis was related to enhancing $\mathrm{Bcl}-\mathrm{xL}$ immunoreactive cells, $\mathrm{Bcl}-2$ expression, $\mathrm{TH}^{+}$neurons, reducing the level of caspase- 3 cells, Bax, TUNEL neurons, and iNOS expression, and preventing c-Jun NH2-terminal kinase (JNK) signaling cascade $[19,20,24,26,30,31]$. Therefore, G-Rgl exerts beneficial effects on multiple aspects of the pathophysiology in PD.

4.4. Implications. It is well known that animal experiments have contributed to our understanding of mechanisms of diseases, but the translation of preclinical experiment which results in a prediction of the effectiveness of treatment strategies in clinical trials is still challenging [46, 47]. Previous studies [13] suggested that the quality of the research design is an important factor affecting the outcome. The main causes for the failure of translation of animal studies to human clinical trials include inadequate animal data and overoptimistic conclusions about efficacy drawn from methodologically flawed animal studies. Thus, it is essential to improve the methodological standards in the design, execution, and reporting of preclinical PD studies in the future.

Quantitative and statistical analysis of Ginsenosides in plasma indicates that PD type exhibits higher concentration and longer half-life than PT type [48]. Due to the low membrane permeability, active biliary excretion, and biotransformation, the oral bioavailability of G-Rg1 is very low [4951]. After an oral administration of G-Rgl, the experiment in rats indicated that the area under the curve of G-Rg1 is $28.93 \mu \mathrm{g} \cdot \mathrm{h} \cdot \mathrm{L}^{-1}$ and the mean value of half-life is 15.26 hours. The peak concentration is $7.15 \mu \mathrm{g} \cdot \mathrm{L}^{-1}$, while $T_{\max }$ is 2.19 hours. In clinic, the use of ginseng for the suggestive symptoms of PD could date back to 1623-1670 AD recorded in Yizong Jiren Bian (Compiled Texts on Self Duty of Medicine) by Gao Gufeng who discussed the pathogenesis of tremor syndrome in the chapter shiver, shake, tremble: "Pathogenesis is mainly due to deficiency of Qi and Blood. The bones and muscles could not get enough nourishment, causing tremble that could not be controlled." Ginseng Tonic Decoction should be used for treatment to invigorate Qi and Blood [5]. In fact, ginseng was one of most commonly used herbs for tremor syndrome from the Han Dynasty to the end of the Qing Dynasty (206 BC-1911 AD) in China by using the frequency statistics according to 232 prescriptions involving 193 herbs and 2529 total frequency of herbs [5]. In modern time, several clinical studies have been conducted to assess the efficacy and safety of ginseng prescription for $\mathrm{PD}$, and the results indicated that ginseng prescriptions could significantly ameliorate the motor symptoms and improve the quality of life [52, 53]. However, no clinical study of G-Rgl for PD has been yet conducted. In the present study, the findings indicated that GRg1 exerted potential neuroprotective functions against PD and its mechanisms are involved with on multiple aspects of the pathophysiology in multiple PD models. Thus, G-Rg1 may be a promising candidate neuroprotectant from bench to bedside. In addition, high-quality randomized controlled trials (RCTs) and a systematic review of those RCTs are commonly regarded the highest level of evidence in judging the treatment efficacy and safety of interventions [54]. Given the huge gap between the animal studies and the clinical trials, seeking and developing innovative neuroprotectants and further rigorous RCTs are urgently needed. In the present study, the findings indicated that G-Rg1 exerted potential neuroprotective functions against $\mathrm{PD}$ and its mechanisms are involved with multiple aspects of the pathophysiology of PD. Thus, G-Rgl may be a promising candidate neuroprotectant from bench to bedside.

\section{Conclusion}

G-Rg1 exerted potential neuroprotective functions against PD despite of the methodological flaws. In addition, we identified an important area, which is worthy of further study. G-Rg1 as a promising clinical candidate neuroprotectant for PD needs to be further confirmed by clinical trials.

\section{Competing Interests}

None of the authors have potential competing interests to be disclosed.

\section{Authors' Contributions}

Liang Song, Meng-Bei Xu, and Xiao-Li Zhou contributed equally to this work.

\section{Acknowledgments}

This project was supported by the grant of the Administration of Traditional Chinese Medicine of Zhejiang Province (2010ZQ010), Wenzhou Municipal Science and Technology Bureau in Zhejiang province (Y20130337), Young and Middle-Aged University Discipline Leaders of Zhejiang Province, China (2013277), and Zhejiang Provincial Program for the Cultivation of High-Level Health Talents (2015).

\section{References}

[1] G. E. Alexander, "Biology of Parkinson's disease: pathogenesis and pathophysiology of a multisystem neurodegenerative disorder," Dialogues in Clinical Neuroscience, vol. 6, no. 3, pp. 259280, 2004.

[2] M. Löhle and H. Reichmann, "Clinical neuroprotection in Parkinson's disease-still waiting for the breakthrough," Journal of the Neurological Sciences, vol. 289, no. 1-2, pp. 104-114, 2010.

[3] P. Calabresi, M. D. Filippo, V. Ghiglieri, N. Tambasco, and B. Picconi, "Levodopa-induced dyskinesias in patients with 
Parkinson's disease: filling the bench-to-bedside gap," The Lancet Neurology, vol. 9, no. 11, pp. 1106-1117, 2010.

[4] T. A. Zesiewicz and M. L. Evatt, "Potential influences of complementary therapy on motor and non-motor complications in parkinsons disease," CNS Drugs, vol. 23, no. 10, pp. 817-835, 2009.

[5] G. Zheng, “Therapeutic History of Parkinson's Disease in Chinese Medical Treatises," The Journal of Alternative and Complementary Medicine, vol. 15, no. 11, pp. 1223-1230, 2009.

[6] S.-Y. Nah, D.-H. Kim, and H. Rhim, "Ginsenosides: are any of them candidates for drugs acting on the central nervous system?” CNS Drug Reviews, vol. 13, no. 4, pp. 381-404, 2007.

[7] J. Lu, Q. Yao, and C. Chen, "Ginseng compounds: an update on their molecular mechanisms and medical applications," Current Vascular Pharmacology, vol. 7, no. 3, pp. 293-302, 2009.

[8] K. Leung and A. Wong, "Pharmacology of ginsenosides: a literature review," Chinese Medicine, vol. 5, no. 1, article no. 20, 2010.

[9] B. Jiang, Z. Xiong, J. Yang et al., "Antidepressant-like effects of ginsenoside Rg1 are due to activation of the BDNF signalling pathway and neurogenesis in the hippocampus," British Journal of Pharmacology, vol. 166, no. 6, pp. 1872-1887, 2012.

[10] H. J. Kim, P. Kim, and C. Y. Shin, "A comprehensive review of the therapeutic and pharmacological effects of ginseng and ginsenosides in central nervous system," Journal of Ginseng Research, vol. 37, no. 1, pp. 8-29, 2013.

[11] K. Radad, G. Gille, L. Liu, and W.-D. Rausch, "Use of ginseng in medicine with emphasis on neurodegenerative disorders," Journal of Pharmacological Sciences, vol. 100, no. 3, pp. 175-186, 2006.

[12] H. B. van der Worp, D. W. Howells, E. S. Sena et al., "Can animal models of disease reliably inform human studies?" PLoS medicine, vol. 7, no. 3, Article ID e1000245, 2010.

[13] M. R. Macleod, T. O'Collins, D. W. Howells, and G. A. Donnan, "Pooling of animal experimental data reveals influence of study design and publication bias," Stroke, vol. 35, no. 5, pp. 1203-1208, 2004.

[14] H. M. Vesterinen, E. S. Sena, K. J. Egan et al., "Meta-analysis of data from animal studies: a practical guide," Journal of Neuroscience Methods, vol. 221, pp. 92-102, 2014.

[15] H. W. E. Zhang, Z. X. I. Lin, C. Xu, C. Leung, and L. S. U. Chan, "Astragalus (a traditional Chinese medicine) for treating chronic kidney disease," The Cochrane Database of Systematic Reviews, vol. 10, Article ID CD008369, 2014.

[16] Y. Heng, Q.-S. Zhang, Z. Mu, J.-F. Hu, Y.-H. Yuan, and N.H. Chen, "Ginsenoside Rg1 attenuates motor impairment and neuroinflammation in the MPTP-probenecid-induced parkinsonism mouse model by targeting $\alpha$-synuclein abnormalities in the substantia nigra," Toxicology Letters, vol. 243, pp. 7-21, 2016.

[17] W. Jiang, Z. Wang, Y. Jiang, M. Lu, and X. Li, "Ginsenoside Rg1 ameliorates motor function in an animal model of Parkinson's disease," Pharmacology, vol. 96, no. 1-2, pp. 25-31, 2015.

[18] T.-T. Zhou, G. Zu, X. Wang et al., "Immunomodulatory and neuroprotective effects of ginsenoside Rg1 in the MPTP(1methyl-4-phenyl-1,2,3,6-tetrahydropyridine) -induced mouse model of Parkinson's disease," International Immunopharmacology, vol. 29, no. 2, pp. 334-343, 2015.

[19] X.-C. Chen, Y.-C. Zhou, Y. Chen, Y.-G. Zhu, F. Fang, and L.M. Chen, "Ginsenoside Rg1 reduces MPTP-induced substantia nigra neuron loss by suppressing oxidative stress," Acta Pharmacologica Sinica, vol. 26, no. 1, pp. 56-62, 2005.
[20] X.-C. Chen, Y. Chen, Y.-G. Zhu, F. Fang, and L.-M. Chen, "Protective effect of ginsenoside Rg1 against MPTP-induced apoptosis in mouse substantia nigra neurons," Acta Pharmacologica Sinica, vol. 23, no. 9, pp. 829-834, 2002.

[21] J. B. Wei, F. Yang, and S. X. Wang, "Influence of Ginsenoside Rg1 on expression of erythropoietin-producing hepatocellular cell line A4 in substantia nigras of mice with Parkinson's disease induced by 1-methy-4-pheny-1,2,3,6-tetrahy-dro-pyrdine," Journal of Xinxiang Medical University, vol. 32, no. 5, pp. 404407, 2015.

[22] S. X. Wang, F. X. Zhu, P. Y. Li, and Y. Duan, "Effects of ginsenoside $\mathrm{Rgl}$ on apoptosis of dopamine neurons, expression 1,TH and P-c-Jun in substantia nigra of mice with Parkinson," Journal of Zhengzhou University, vol. 50, no. 2, pp. 176-180, 2015.

[23] F. X. Zhu, Y. Duan, P. Y. Li, and S. X. Wang, "Effect of ginsenoside Rg1 on the expressions of tyrosine hydroxylase, ephrinB2 and phosphorylated c-Jun in substantia nigra of mice with Parkinson's disease," Journal of Xinxiang Medical University, vol. 31, no. 10, pp. 781-785, 2014.

[24] Q. Wang, H. Zhang, M. Liu et al., "Influence of ginsenoside Rg1 in expressions of FADD and FLIP in substantia nigra of Parkinson's disease model mice," Journal of Jilin University Medicine Edition, vol. 40, no. 5, pp. 962-966, 2014.

[25] Z. Yan, L. Wu, D. D. Xue, X. Q. Gao, and W. F. Chen, "Effects of Gesenoside Rgl and insulin-like growth factor-I on dopaminergic neurons in Parkinson's disease mice," Acta Academic Medicine QingDao University, vol. 50, no. 4, pp. 283285, 2014.

[26] J. L. R. Liu, L. Li, Y. X. Zhang, and Z. F. Zhang, “The protective effect of Ginsenoside Rg1 on nerves in the mice model of Parkinson's disease," Clinical Medicine of China, vol. 24, no. 9, pp. 898-900, 2008.

[27] G. J. Yang, J. P. Zhang, and Q. Y. Li, "Effect of ginsenoside Rg1 on neural stem cells in subventricular zone in modeled mice of Parkinson's disease," Sichuan Journal of Physiological Sciences, vol. 31, no. 1, pp. 17-19, 2009.

[28] G. Z. Ji, The experimental research for evaluating mice's dopaminergic neurons function by $99 m$ TC-TRODAT-1 [Postgraduates' degree Thesis], 2008.

[29] Q. Wang, H. Zheng, Z.-F. Zhang, and Y.-X. Zhang, "Ginsenoside Rg1 modulates COX-2 expression in the substantia nigra of mice with MPTP-induced Parkinson disease through the P38 signaling pathway," Journal of Southern Medical University, vol. 28, no. 9, pp. 1594-1598, 2008.

[30] H. D. Yang, Neurprotective effects of Ginsenoside Rg1 on the dopaminergic neuron loss in the substantia nigra of MPTPtreated C57BL6 mice [Postgraduates' degree Thesis], 2007.

[31] Y. C. Zhou, Y. G. Zhu, F. Fang, and L. M. Chen, “The influence of Ginsenoside Rg1 on JNK signaling cascade in PD mouse substantia nigra neurons," Acta Anatomica Sinica, vol. 34, no. 5, pp. 477-481, 2003.

[32] G. G. Meng, Z. F. Zhang, X. J. Huan, Z. F. Wei, R. Li, and Y. $X$. Zhang, "Ginsenside Rgl protects the dopaminergic neurolls in substantia nigra against the apoptosis via downregulate the endoplasmic reticulum stress proteins expressions in the MPTP-induced Parkinson's disease mouse model," Chinese Journal of Anatomy, vol. 34, no. 3, pp. 358-361, 2011.

[33] F. W. Yang, Influence of Ginsenside Rg1 on expressions of EphB6 and ephrinB1 in substantia nigra of mice with Parkinson's disease induced by MPTP [Postgraduates' degree Thesis], 2014.

[34] Y. S. Wang, Y. X. Zhang, S. P. Wei, Z. F. Zhang, and Q. Y. Tian, "Influence of Ginsenoside Rg1 on p-c-Jun and COX-2 
expression insubstantia nigra of the MPTP mouse model of subacute Parkinson's disease," Chinese Journal of Neuroanatomy, vol. 25, no. 4, pp. 432-436, 2009.

[35] L. Xu, W. Chen, and M. Wong, "Ginsenoside Rg1 protects dopaminergic neurons in a rat model of Parkinson's disease through the IGF-I receptor signalling pathway," British Journal of Pharmacology, vol. 158, no. 3, pp. 738-748, 2009.

[36] J. Jie, Study of neuroprotective effects of antioxidant Ginsenoside Rg1 on the Parkinson rats [Postgraduates' degree Thesis], 2010.

[37] L. Xu, L.-X. Liu, and W.-F. Chen, "Effect and mechanism on dopamine contents of striatum in rat model of Parkinson's disease ginsenoside Rgl," Journal of Chinese Materia Medica, vol. 33, no. 15, pp. 1856-1859, 2008.

[38] L. Z. M. Xu and W. F. Chen, "Neuroprotective effect of ginsenoside Rg1 on dopaminergic neurons and blocking effect of ICI182, 780," Journal of Shanxi Medical University, vol. 38, no. 8, pp. 677-681, 2007.

[39] T. Zhou, G. Zu, X. Zhang et al., "Neuroprotective effects of ginsenoside $\mathrm{Rg} 1$ through the Wnt/ $\beta$-catenin signaling pathway in both in vivo and in vitro models of Parkinson's disease," Neuropharmacology, vol. 101, pp. 480-489, 2016.

[40] X.-C. Sun, X.-F. Ren, L. Chen, X.-Q. Gao, J.-X. Xie, and W.F. Chen, "Glucocorticoid receptor is involved in the neuroprotective effect of ginsenoside Rg1 against inflammationinduced dopaminergic neuronal degeneration in substantia nigra," Journal of Steroid Biochemistry and Molecular Biology, vol. 155, pp. 94-103, 2016.

[41] G. H. Guyatt, A. D. Oxman, V. Montori et al., "GRADE guidelines: 5. Rating the quality of evidence-publication bias," Journal of Clinical Epidemiology, vol. 64, no. 12, pp. 1277-1282, 2011.

[42] E. D. M. Rooke, H. M. Vesterinen, E. S. Sena, K. J. Egan, and M. R. Macleod, "Dopamine agonists in animal models of Parkinson's disease: a systematic review and meta-analysis," Parkinsonism and Related Disorders, vol. 17, no. 5, pp. 313-320, 2011.

[43] B. C. Kahan, S. Cro, C. J. Doré et al., "Reducing bias in openlabel trials where blinded outcome assessment is not feasible: strategies from two randomised trials," Trials, vol. 15, no. 1, article no. 456, 2014.

[44] U. Held, "On the impact of sample size calculation and power in clinical research," Praxis, vol. 103, no. 20, pp. 1197-1201, 2014.

[45] K. F. Schulz and D. A. Grimes, "Sample size calculations in randomised trials: mandatory and mystical," The Lancet, vol. 365, no. 9467, pp. 1348-1353, 2005.

[46] D. G. Hackam, "Translating animal research into clinical benefit," British Medical Journal (Clinical Research Edition), vol. 334, no. 7586, pp. 163-164, 2007.

[47] D. G. Hackam and D. A. Redelmeier, "Translation of research evidence from animals to humans," JAMA, vol. 296, no. 14, pp. 1727-1732, 2006.

[48] Y. Takino, "Studies on the pharmacodynamics of ginsenosideRg1, -Rb1 and -Rb2 in rats," Yakugaku Zasshi, vol. 114, no. 8, pp. 550-564, 1994.

[49] Y. Zhang, L. Lin, G. Y. Liu, J. X. Liu, and T. Li, "Pharmacokinetics and brain distribution of ginsenosides after administration of Sailuotong," China Journal of Chinese Materia Medica, vol. 39, no. 2, pp. 316-321, 2014 (Chinese).

[50] H. Liu, J. Yang, F. Du et al., "Absorption and disposition of ginsenosides after oral administration of panax notoginseng extract to rats," Drug Metabolism and Disposition, vol. 37, no. 12, pp. 2290-2298, 2009.
[51] M. Karikura, T. Miyase, H. Tanizawa, Y. Takino, T. Taniyama, and T. Hayashi, "Studies on absorption, distribution, excretion and metabolism of Ginseng saponins. V. The decomposition products of ginsenoside $\mathrm{Rb}_{2}$ in the large intestine of rats," Chemical and Pharmaceutical Bulletin, vol. 38, no. 10, pp. 28592861, 1990.

[52] X. X. Wen, "Treating 68 cases of tremble of Qixue Kuixu type with the Renshen Yangrong decoction," Clinical Journal of Chinese Medicine, vol. 5, no. 3, p. 67, 2013.

[53] D. G. Wang, "Clinical observation of Ginseng Guipi decoction on elderly patients of deficiency of qi and blood type with Parkinson's disease," Guiding Journal of Traditional Chinese Medicine and Pharmacy, vol. 20, no. 6, pp. 126-127, 2014.

[54] J. Wei, W. Yang, S. Yin, C. Wang, Y. Wang, and G. Zheng, "The quality of reporting of randomized controlled trials of electroacupuncture for stroke," BMC Complementary and Alternative Medicine, vol. 16, no. 1, article no. 512, 2016. 


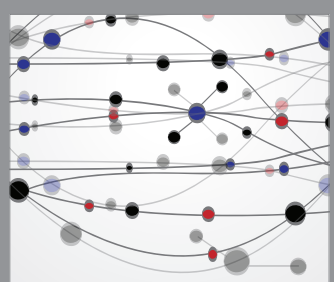

The Scientific World Journal
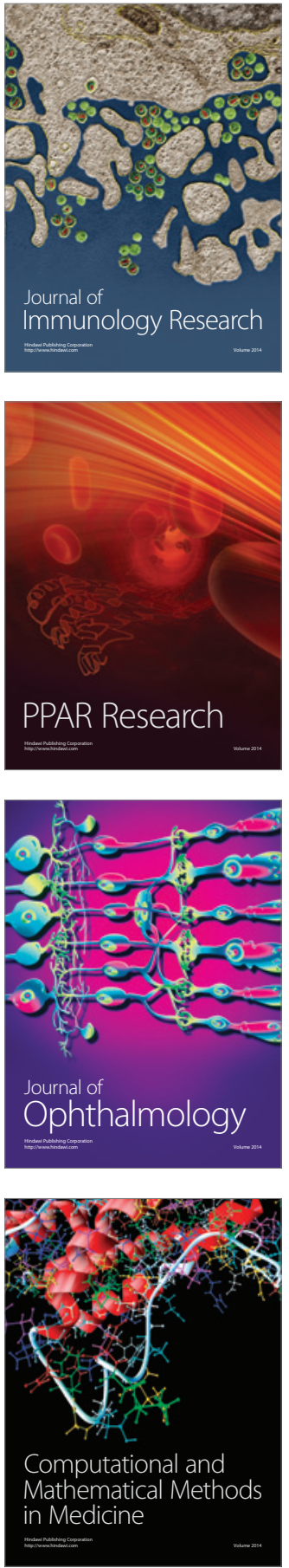

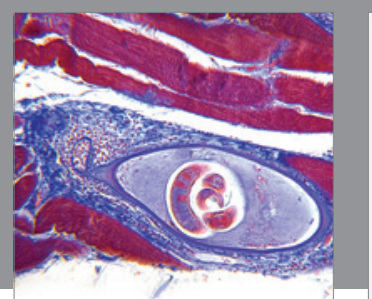

Gastroenterology Research and Practice
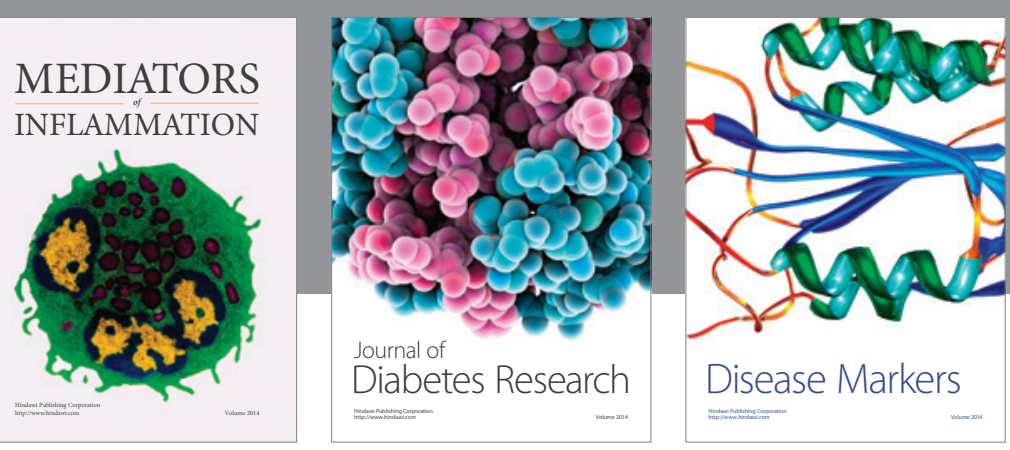

Disease Markers

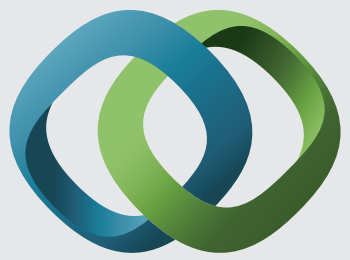

\section{Hindawi}

Submit your manuscripts at

https://www.hindawi.com
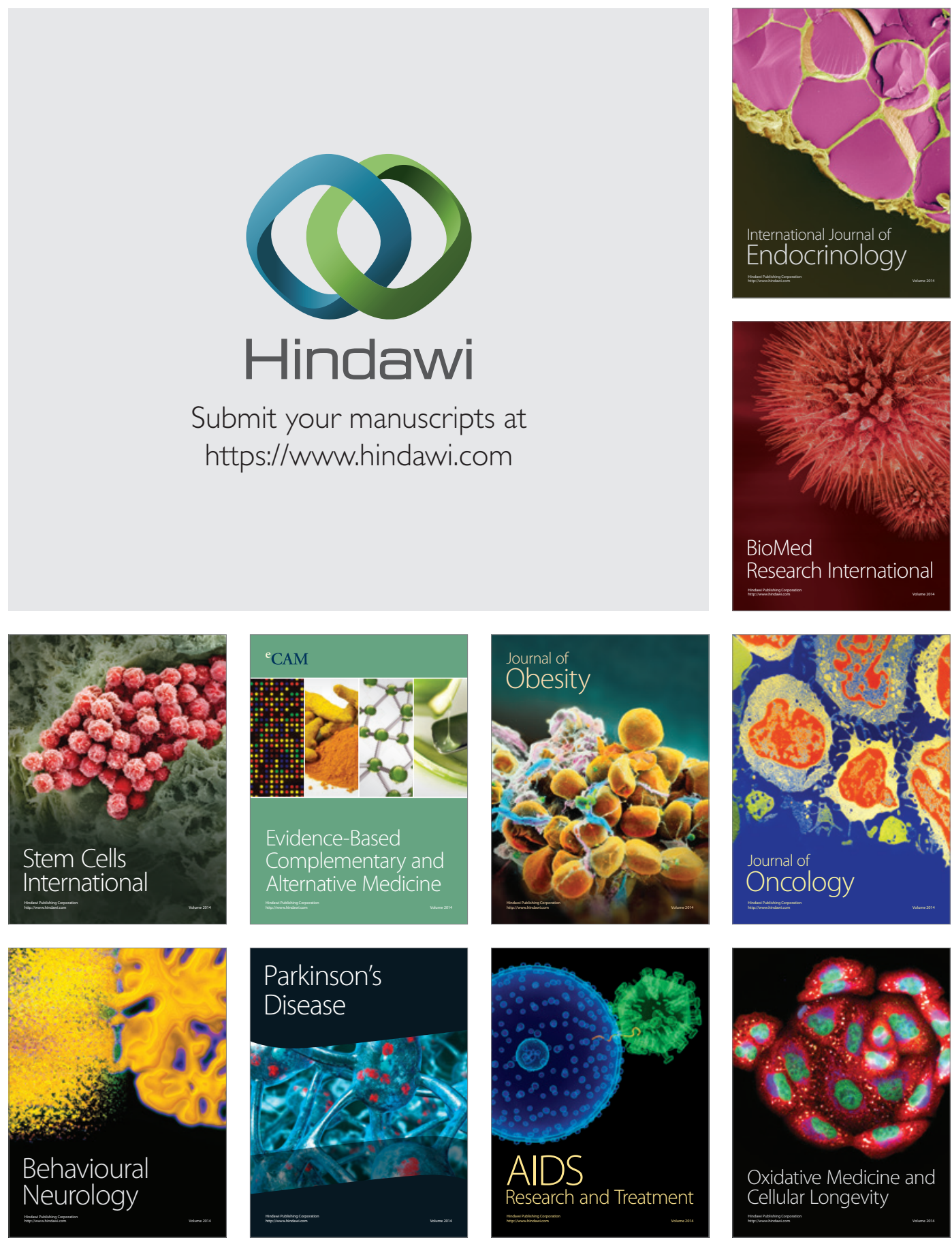